ARTICLE

\title{
Engineering and elucidation of the lipoinitiation process in nonribosomal peptide biosynthesis
}

Lin Zhong (1,3, Xiaotong Diao (10 1,3, Na Zhang ${ }^{1,3}$, Fengwei Li ${ }^{1}$, Haibo Zhou ${ }^{1}$, Hanna Chen ${ }^{1}$, Xianping Bai ${ }^{1}$, Xintong Ren (1) ${ }^{1}$, Youming Zhang ${ }^{1 凶}$, Dalei Wu $\mathbb{0}^{1,2 凶} \&$ Xiaoying Bian $\mathbb{D}^{1 凶}$

Nonribosomal peptide synthetases containing starter condensation domains direct the biosynthesis of nonribosomal lipopeptides, which generally exhibit wide bioactivities. The acyl chain has strong impacts on bioactivity and toxicity, but the lack of an in-depth understanding of starter condensation domain-mediated lipoinitiation limits the bioengineering of NRPSs to obtain novel derivatives with desired acyl chains. Here, we show that the acyl chains of the lipopeptides rhizomide, holrhizin, and glidobactin were modified by engineering the starter condensation domain, suggesting a workable approach to change the acyl chain. Based on the structure of the mutated starter condensation domain of rhizomide biosynthetic enzyme RzmA in complex with octanoyl-CoA and related point mutation experiments, we identify a set of residues responsible for the selectivity of substrate acyl chains and extend the acyl chains from acetyl to palmitoyl. Furthermore, we illustrate three possible conformational states of starter condensation domains during the reaction cycle of the lipoinitiation process. Our studies provide further insights into the mechanism of lipoinitiation and the engineering of nonribosomal peptide synthetases.

\footnotetext{
${ }^{1}$ Helmholtz International Lab for Anti-Infectives, Shandong University-Helmholtz Institute of Biotechnology, State Key Laboratory of Microbial Technology, Shandong University, Qingdao, Shandong 266237, China. ${ }^{2}$ Suzhou Research Institute, Shandong University, Suzhou, Jiangsu 215123, China. ${ }^{3}$ These authors contributed equally: Lin Zhong, Xiaotong Diao, Na Zhang. ${ }^{凶}$ email: zhangyouming@sdu.edu.cn; dlwu@sdu.edu.cn; bianxiaoying@sdu.edu.cn
} 
$\mathrm{N}$ onribosomal peptide synthetases (NRPSs) are multimodular enzymes that catalyze the biosynthesis of diverse peptides with a wide variety of activities ${ }^{1,2}$. Each NRPS module, which typically includes a condensation (C) domain, an adenylation (A) domain, and a peptidyl carrier protein (PCP) domain, incorporates a single amino acid into a growing peptide chain, forming the peptidyl backbone by sequential condensations $^{3}$. The nonribosomal lipopeptides represented by the last resort antibiotics daptomycin and polymyxin, featuring an acyl tail on the N-terminus of the linear or cyclic peptidyl backbone, are typical products of NRPSs ${ }^{4-9}$. Because the N-terminal acyls are highly diverse, ranging from short acetyl to long fatty acyl groups, we here mention the NRPS-derived peptide with an Nacylation as a nonribosomal lipopeptide for the purpose of a convenient description. The incorporation of an acyl into peptidyl backbone is usually implemented by a starter condensation (Cs) domain, normally located at the beginning of the initial NRPS module, via the formation of an amide bond to the $\alpha$-amino group of the first amino acid residue, a process also known as lipoinitiation ${ }^{10}$. The acyl moiety is delivered to the Cs domain either by tethering to an acyl carrier protein (ACP), as in the cases of calcium-dependent antibiotics (CDAs) and lipopeptide A54145 $5^{11,12}$, or simply in a free acyl-CoA form, as in the cases of surfactin and glidobactin ${ }^{13,14}$ (Fig. 1a). The N-terminal acyl chains of lipopeptides strongly affect the biological activity and toxicity of the lipopeptides ${ }^{4,15-17}$. Thus, the engineering of NRPS machinery (especially the Cs domain) to produce lipopeptides with a desired acyl chain is of great interest, and its success depends heavily on our understanding of the specificity and mechanism of lipoinitiation.

The mechanism of the lipoinitiation reaction has been investigated by in vitro biochemical experiments using the dissected Cs domains or entire initiation modules (Cs-A-PCP), respectively, from surfactin synthetase SrfAA, glidobactin synthetase GlbF, CDA synthetase CdaPS1, and A54145 synthetase LptA, revealing the substrate specificity of Cs domains for fatty acyl moieties ${ }^{11-14}$ The crystal structure of the dissected Cs domain from CdaPS1 enabled researchers to propose an active-site tunnel for the accommodation of both "donor" and "acceptor" substrates ${ }^{18}$. Due to the low affinity of $\mathrm{C}$ domains for substrate analogs, a covalent mimic of an acceptor substrate was adopted to obtain the cocrystal structure of CdaPS1-Cs, which revealed the role of second histidine (of the "HHxxxD" motif) in substrate positioning and the acceptor substrate specificity of the condensation reaction ${ }^{19}$. Although a number of NRPS structures involving the $\mathrm{C}$ domains have been solved as single-domain ${ }^{18-20}$ or a

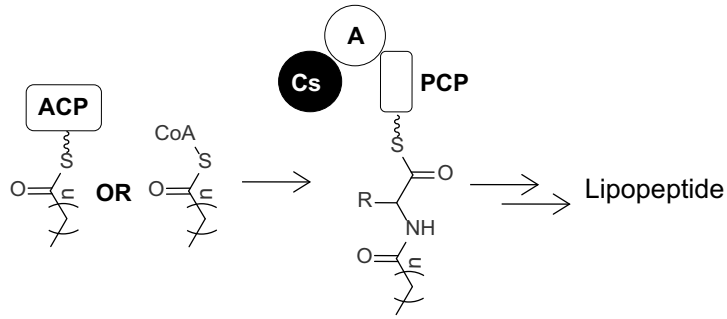

b

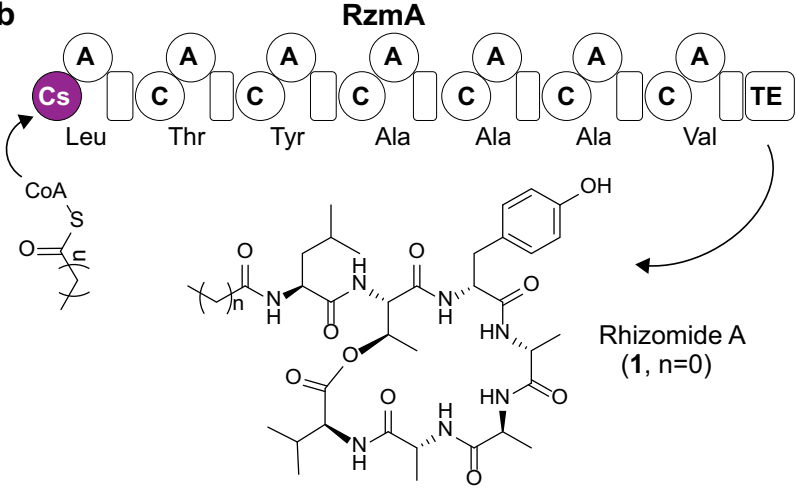

C

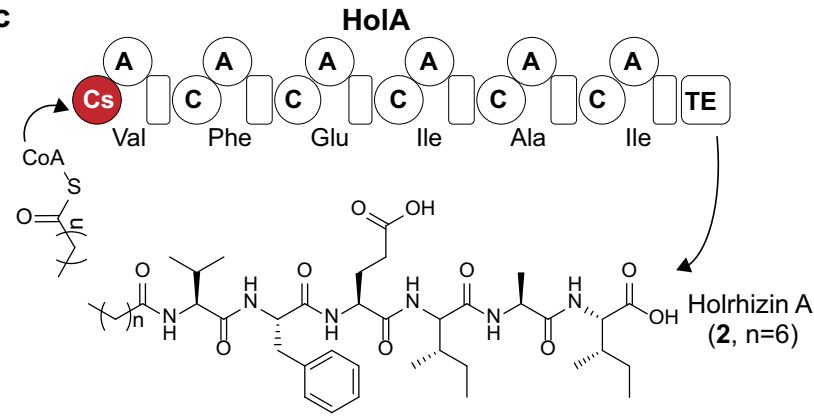

d

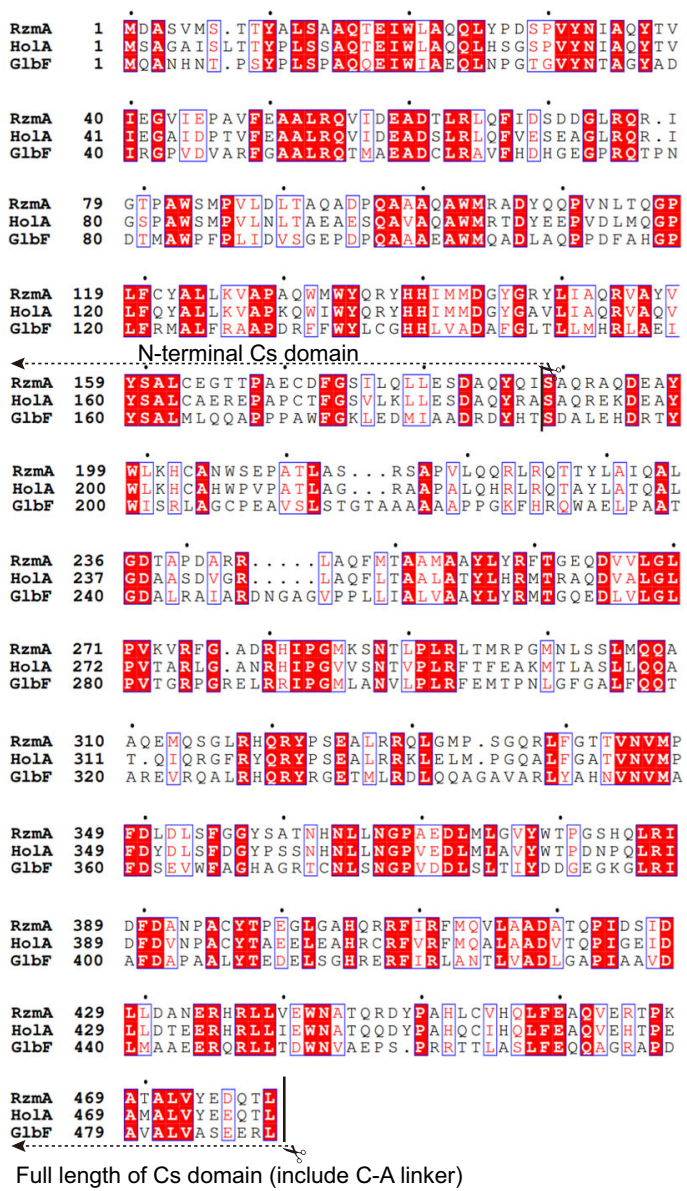

Fig. 1 Biosynthesis of nonribosomal lipopeptides. a The starter condensation (Cs) domain catalyzes the lipoinitiation of lipopeptide biosynthesis using acyl-ACP or acyl-CoA as a substrate. b, c The rhizomide and holrhizin biosynthetic pathways (RzmA and HolA) from P. rhizoxinica, and their main products rhizomide A (1) and holrhizin A (2), which bear a short acetyl and a medium octanoyl moieties, respectively. d Sequence alignment of RzmA-Cs, HolA-Cs, and GlbF-Cs. The swapping sites of the full-length $\mathrm{Cs}$ domain and subdomain ( $\mathrm{N}$-lobe) are labeled with a black line and a pair of scissors, respectively. A adenylation domain, ACP acyl carrier protein, C condensation domain, PCP peptidyl carrier protein, TE thioesterase domain. 
multidomain (even modular) structures ${ }^{21-28}$, CdaPS1-Cs remains the only lipoinitiation-conducting $\mathrm{Cs}$ domain with a reported structure. Moreover, very few $\mathrm{C}$ domain structures were solved in complex with substrates; in particular, none were solved with the acyl moiety. These setbacks obstruct the elucidation of the lipoinitiation mechanism and engineering of the acyl chain of lipopeptides.

Two types of lipopeptides, rhizomide A (1) and holrhizin A (2), with different acyl chains (Fig. 1b, c), were discovered in the bacterium Paraburkholderia rhizoxinica HKI 454 in our previous work $^{29-31}$. Their synthetases possess Cs domains with a high sequence identity. However, the lengths of acyl chains they normally load are quite different (C2 vs C8), making this pair of lipopeptides an ideal model to investigate donor substrate specificity.

In this work, we successfully change the acyl chains of both rhizomide and holrhizin by swapping the Cs domain or mutation of a key amino acid residue, and obtain a Cs mutant with stronger binding to the acyl-CoA substrate. Then, we solve multiple cocrystal structures of RzmA-Cs mutants with donor and/or acceptor substrates. The co-complex with octanoyl-CoA (C8$\mathrm{CoA}$ ) reveals a binding pocket within RzmA-Cs to determine the selectivity of lipid chains, and donor substrate is extended up to palmitoyl-CoA (C16) from native acetyl-CoA (C2) through additional point mutations in vivo and in vitro. In addition to substrate specificity, these unliganded and co-complex structures of RzmA-Cs facilitate our understanding of other aspects of lipoinitiation, including the confirmed substrate positioning function of the second histidine in "HHxxxDG" motif for both "donor" and "acceptor" substrates, and more importantly the potential conformational changes that Cs domains (and likely also other C domains) would undertake throughout the condensation reaction cycle.

\section{Results}

Changing the acyl chains by Cs (sub)domain swapping. Genome mining of two Cs domain-embedded cryptic NRPS biosynthetic gene clusters, $r z m A$ and holA, in the bacterium $P$. rhizoxinica HKI 454 resulted in the discovery of two distinct types of lipopeptides, cyclic rhizomide A (1) containing an uncommon acetyl chain (C2) (Fig. 1b) and linear holrhizin A (2) harboring a medium octanoyl (C8) chain (Fig. 1c) ${ }^{29-31}$. Rhizomide A and holrhizin A differ in the length of acyl chains, suggesting that their Cs domains may be selective for different acyl substrates. Within the rzmA and holA biosynthetic gene clusters, no putative gene homologous to those encoding ACP or fatty acyl ligase (AL) was identified. Thus, we infer that the Cs domains of RzmA and HolA may catalyze lipoinitiation using acyl-CoAs as direct substrates, but the possibility of harnessing acyl-ACPs as direct substrates cannot be fully excluded, because the Cs domains might capture acyl-ACPs from the primary metabolism of bacteria. The in vitro biochemical reactions of dissected RzmACs and HolA-Cs domains proved that they could catalyze the condensation between the acyl-CoAs (3a and 3d) and corresponding first amino acid mimics, namely, aminoacyl-Nacetylcysteamine thioesters (aminoacyl-SNACs, 4 and 6$)^{32}$ (Supplementary Fig. 1).

Although RzmA-Cs and HolA-Cs domains share a high sequence identity (73\%, Fig. 1d), they showed totally different selections of donor acyl substrates in vitro and in vivo, furnishing a possible strategy to change the acyl chains of lipopeptides by the genetic engineering of Cs domains. We conducted full-length $\mathrm{Cs}$ domain (including $\mathrm{C}-\mathrm{A}$ linker) and subdomain (N-lobe) swapping experiments in the rzmA and holA gene clusters by exchanging the coding regions of two Cs (sub)domains, and transferred them into the heterologous host Schlegelella brevitalea strain DSM 7029 for functional expression (Figs. 1d, 2a-d) 33,34 . As expected, rhizomide derivative 1d (Fig. $1 \mathrm{~b}(n=4)$, Fig. 2e) with an octanoyl (C8) chain and a holrhizin derivative 2a (Fig. 1c $(n=0)$, Fig. 2f) with an acetyl (C2) chain were produced with decent yields, and their structures were confirmed by ultraperformance liquid chromatography coupled with highresolution tandem mass spectrometry (UPLC-HRMS/MS, Supplementary Figs. 2 and 3) and nuclear magnetic resonance (NMR, Fig. 2e, f, Supplementary Tables 1-3 and Supplementary Figs. 1120), respectively. The subdomain (N-terminal or N-lobe) swapping of the Cs domain also generated the expected products (1d and $\mathbf{2 a}$ ) but in a relatively lower yield and conversion ratio (Fig. 2c, d), suggesting that Cs domain swapping would be a feasible approach to change the acyl chains of lipopeptides.

To further test this approach, we also performed Cs (sub) domain swapping in GlbF of the glidobactin biosynthetic pathway in the original producer S. brevitalea DSM 702935. The full-length GlbF-Cs domain was replaced by RzmA-Cs, HolA-Cs, as well as GlpC-Cs domain derived from the glidopeptin synthetase GlpC, which incorporates a decanoyl (C10) chain ${ }^{29}$. The exchange of HolA-Cs and Glp-Cs changed the original unsaturated 2(E),4(E) dodecadienoyl of glidobactin A to saturated octanoyl (C8) and decanoyl (C10) chains with improved yields (Fig. 3a, b, NMR: Supplementary Table 4, Supplementary Figs. 29-32), respectively. However, the change to RzmA-Cs failed to produce any products, possible due to the acceptor specificity of the RzmA-Cs domain and/or the donor specificity of the downstream $\mathrm{C}$ domains. The acceptor specificities of the three Cs domains were tested in vitro (Fig. 3c, d), and the RzmA-Cs and HolA-Cs showed relatively broad specificities to the tested acceptor substrates including Thr, indicating that the acceptor specificity of the RzmA-Cs domain failed to affect the swapped glidobactin product. Thus, the reason for the failure could be donor specificity of the downstream $\mathrm{C}$ domain. The relatively broad specificities of RzmA-Cs and HolACs to acceptor substrates explain why the aforementioned Cs domain swapping led to the successful production of acylchanged rhizomide, holrhizin, and glidobactin derivatives, and support the very recent study that novel nonribosomal peptides can be generated by the substitution of A domains alone ${ }^{36}$. Subdomain (N-lobe) swapping of GlbF-Cs with that of GlpC-Cs was also conducted, but no expected compounds were detected. Combined with the swapping in RzmA and HolA (Fig. 2), we propose that the full-length Cs domain swapping could be a worthy choice to modify the acyl chains of lipopeptides, at least for these three lipopeptides.

A key site for controlling of the length of the acyl chain. Next, we tried to determine how the highly similar RzmA-Cs and HolA-Cs domains recognize and select acyl-CoAs with different lengths. The alignment of protein sequences and the construction of a 3D homology model using the structure of CdaPS1-Cs ${ }^{18}$ as a template allowed us to examine those residues that are not conserved in two proteins along the model active-site tunnel (Supplementary Fig. 4). Among a small group of candidate residues, R148 of RzmA (corresponding to A149 of HolA and M165 of CdaPS1) caught our attention. This residue not only sits near the proposed binding pocket for the 2,3-epoxyhexanoyl chain of CDA in the CdaPS1 structure ${ }^{18}$, but also fits well with the simple logic that a longer side-chain of pocket residue would correlate with a shorter substrate chain, and vice versa (i.e., R148 with C2, M165 with C6, and A149 with C8) (Fig. 4a). Therefore, we generated a series of point mutations at this position in rzmA and holA to test whether the selectivity of acyl chains would be changed in vivo (Fig. 4b). We found that compared with 
a

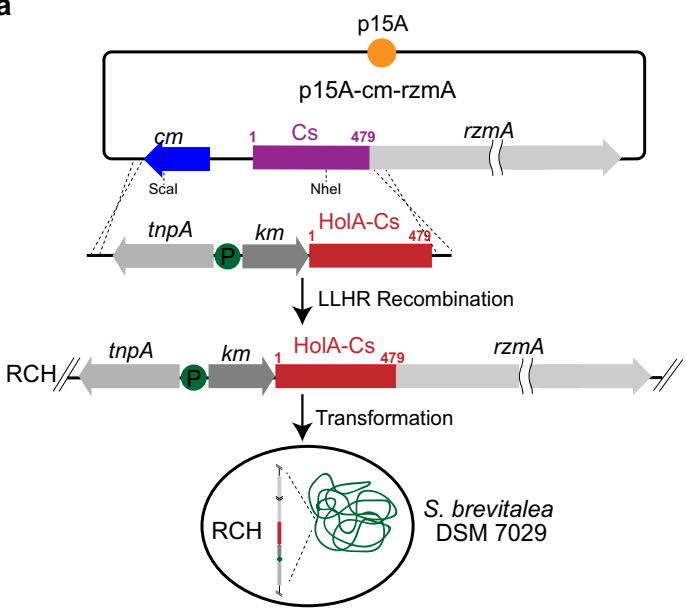

c
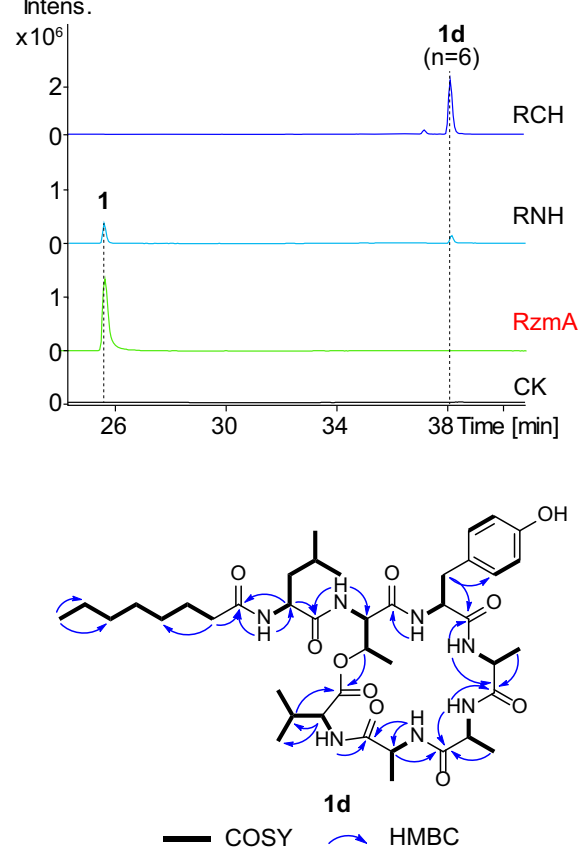

b

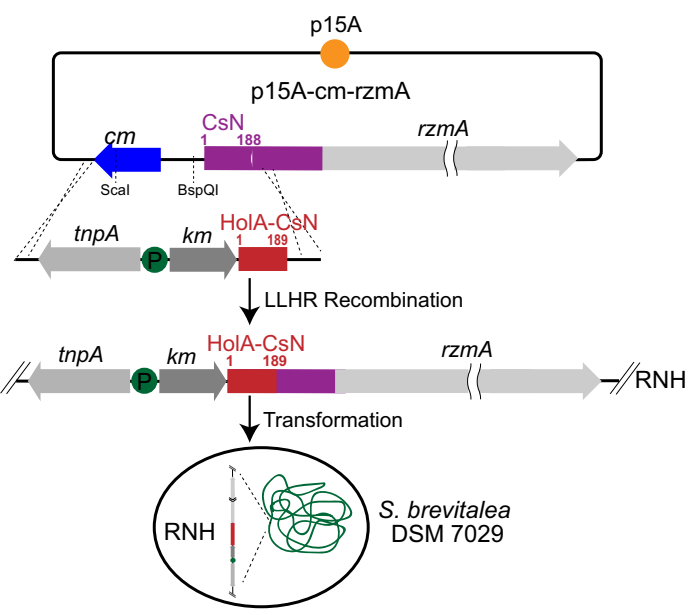

d

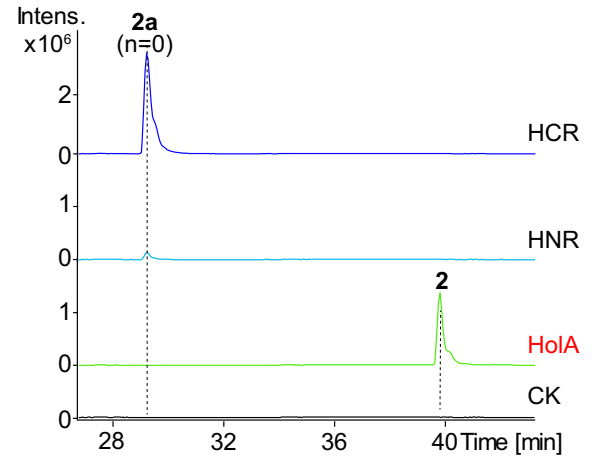

$\mathbf{f}$

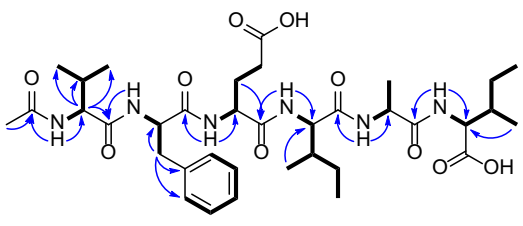

2a

$-\cos \frown \mathrm{HMBC}$

Fig. 2 Exchanging the acyl chains of nonribosomal lipopeptides by $\mathbf{C s}$ (sub)domain swapping. a, b Swapping strategy for full-length $\mathrm{Cs}$ domain (RCH, a) and $\mathrm{N}$-terminal $\mathrm{Cs}$ domain $(\mathrm{RNH}, \mathbf{b})$ of RzmA with those of HolA using recombineering in $E$. coli ${ }^{46}$. The resulting constructs (RCH and RNH) were transformed into S. brevitalea DSM 7029 for heterologous expression. The Cs (sub)domain swapping of HolA with those of RzmA (HCR and HNR) was conducted in the same way. c, d Comparative production analysis of full-length and N-terminal Cs domain swapping of RzmA (c) and HolA (d) showed an exchange of the acyl chains with different efficiencies. Extracted ion chromatograms $(E I C s)$ at $m / z=732.391\left(\left[M+\mathrm{H}^{+}, \mathbf{1}\right)\right.$ and $816.483\left([M+H]^{+}\right.$, 1d) for $(\mathbf{c})$, and at $m / z=817.503\left([\mathrm{M}+\mathrm{H}]^{+}, \mathbf{2}\right)$ and $733.412\left([\mathrm{M}+\mathrm{H}]^{+}, \mathbf{2 a}\right)$ for $(\mathbf{d})$. CK represents the wild type of S. brevitalea DSM 7029 . e, $\mathbf{f}$ Structures and key COSY and HMBC correlations of $\mathbf{1 d}$ and $\mathbf{2 a}$. tnpA transposase gene, $\mathrm{km}$ kanamycin resistance gene, cm chloramphenicol resistance gene, $\mathrm{P}$ promotor. LLHR, linear plus linear homologous recombination. Full information was given in "Methods" section and Supplementary files.

wild-type (WT) RzmA, mutations at R148 indeed altered the production profile of rhizomide in vivo (Fig. 4c). As the residue side chains became shorter $(\mathrm{R} \rightarrow \mathrm{M} \rightarrow \mathrm{L} \rightarrow \mathrm{V} \rightarrow \mathrm{A} \rightarrow \mathrm{G})$, the acyl chains of rhizomides grew longer from $\mathrm{C} 2$ to $\mathrm{C} 8$ or $\mathrm{C} 10$ (Fig. $4 \mathrm{c}$ ). The in vitro substrate competition experiments of excised RzmACs domain variants containing point mutations at R148 using a mixture of equal concentrations of $\mathrm{C} 2-\mathrm{C} 18$ acyl-CoAs as substrates also showed similar alteration of the substrate bias of each mutant (Supplementary Fig. 1e), basically in coherence with our in vivo experiments. A similar trend, but in the reverse direction could be seen for the HolA A149 mutations, in which longer residue side chains led to shorter acyl chains (Fig. 4d). These synchronized changes showed that the length of the side-chain of this key amino acid (R148 in RzmA-Cs, A149 in HolA-Cs) might control the length of acyl chains. The side-chain may act as an entry gate to a potential pocket. Therefore, truncating the sidechain of this residue could give a correspondingly deeper space for the extension of longer acyl-CoAs. These results clearly highlight the important role of residues at this position of Cs domains, in defining the length of acyl chains during lipopeptide biosynthesis.

Co-complex structure-guided engineering of the Cs domain. In addition to the above finding of RzmA R148 (HolA A149), there are still many unanswered questions about lipoinitiation, such as whether more residues are involved in the recognition of substrate acyl chains and whether the production of lipopeptides 
a

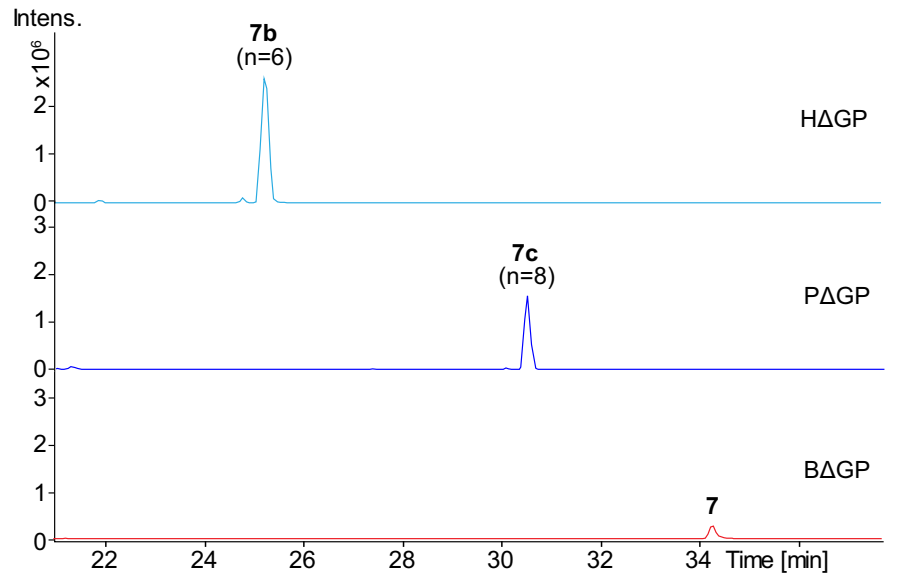

b

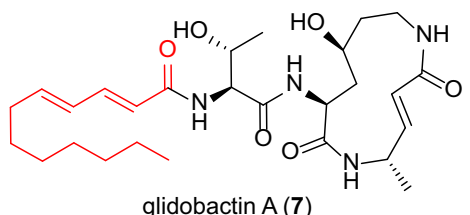

glidobactin A (7)<smiles>C[C@@H](CC(C)(C)C)NC(=O)[C@@H](C[C@@H](O)CCNC(=O)/C=C/[C@@H](C)NC(=O)CC(C)(C)C)NC(=O)[C@@H](NC(=O)CC(C)(C)C)[C@@H](C)O</smiles>

C8-glidobactin A (7b, $n=6)$

C10-glidobactin A (7c, $n=8)$ c<smiles>[R]C(N)[C@H](N)C(=O)SCCNC(C)=O</smiles>

Acyl-Aminoacyl-SNAC d

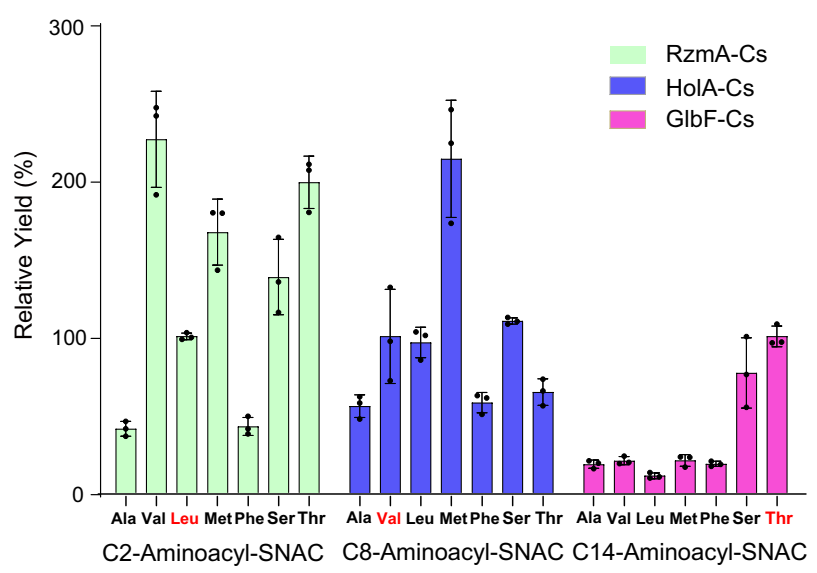

Fig. 3 Cs domain swapping experiments on the glidobactin synthase GlbF and in vitro assay of Cs specificity for acceptor substrates. $\mathbf{a}$, $\mathbf{b}$ Production analysis of full-length domain swapping of GlbF-Cs (B $\Delta$ GP) with HolA-Cs (H $\Delta$ GP) and GlpC-Cs (P $\Delta$ GP) in S. brevitalea DSM 7029 showed an exchange of the acyl chains with different yields. EICs at $m / z=521.333\left([M+H]^{+}, \mathbf{7}\right)$ for $7029 B \Delta G P$, and $469.3021\left([M+H]^{+}, 7 \mathbf{b}\right)$ for $7029 H \Delta G P$, and at $m / z=$ $497.333\left([\mathrm{M}+\mathrm{H}]^{+}, \mathbf{7 c}\right)$ for 7029P $\Delta$ GP. c, $\mathbf{d}$ In vitro assay of $\mathrm{Cs}$ specificity for acceptor substrates. The final products were quantified by comparison with the EIC peak area of native SNACs (100\%), i.e., for RzmA-Cs, every set of tests was quantified with products of L-Leu-SNAC. The native SNACs are marked in red. Experiments were conducted in triplicates $(n=3)$, data are presented as mean values $+/-\mathrm{SD}$, and detailed information is given in the "Methods" section.

with longer chains $(>\mathrm{C} 8)$ is also possible by rational engineering. For this purpose, we sought structural information on these proteins. Despite our successful purification of the dissected Cs domains of both RzmA and HolA, we were able to obtain crystals only for RzmA-Cs, and solved its structure at $2.3 \AA$ in the $P 1$ space group (Supplementary Table 5). Similar to other C domain structures reported previously ${ }^{18,20,25,26}$, RzmA-Cs adopts a "V-shaped" configuration and consists of two subdomains (Nand C-lobes) each representing the chloramphenicol acetyltransferase (CAT) fold (Fig. 5a). Interestingly, in each asymmetric unit there are four molecules of RzmA-Cs with two different conformations (Fig. 5b). The overall structures of the two conformations are quite similar ( $\mathrm{RMSD}=1.52 \AA$ for $\mathrm{Ca}$ ), especially when the two lobes are compared separately $(\mathrm{RMSD}=0.68 \AA$ and $1.30 \AA$ for $\mathrm{C} \alpha$ of the $\mathrm{N}$ - and C-lobes). The major difference comes from the relative positions of the two lobes (i.e., opening angles of the "V" as shown in Fig. 5c). It has been known that the "openness" of C domains can vary in different NRPSs (Fig. $5 \mathrm{~d})^{18}$. However, it is quite unique that here the RzmA-Cs presents two conformations at the same time, emphasizing its intrinsic flexibility before engaging the substrates.

To delineate the interactions between the Cs domain and acylCoA, we wanted to solve their co-complex structure. After our initial attempts to cocrystallize WT RzmA-Cs with acyl-CoAs of various lengths failed, we noticed that the R148A mutant protein showed strong binding to $\mathrm{C} 8-\mathrm{CoA}$, as evidenced by the high $\Delta \mathrm{Tm}$ in protein thermal shift assays (Supplementary Fig. 5) and a $K_{\mathrm{d}}$ value of $\sim 300 \mathrm{nM}$ measured using isothermal titration calorimetry (ITC) (Fig. 6a). Thus we selected this mutant for crystallization, and successfully obtained both unbound and C8-CoAbound structures (Fig. $6 \mathrm{~b}$ and Supplementary Table 5). The unbound R148A structure is mostly identical to that of WT RzmA-Cs in two conformations (Supplementary Fig. 6a). However, the C8-CoA-bound R148A structure shows only one conformation (in the $P 2_{1} 2_{1} 2_{1}$ space group), which is "tighter" than both conformations of the unbound structure (Fig. 6c), indicating that the two lobes of RzmA-Cs may close up to bind acyl-CoA substrates.

This co-complex structure revealed the detailed interactions between the RzmA-Cs R148A mutant and C8-CoA (Fig. 6b-d). The CoA portion binds at the "donor" site of Cs with its loading arm inserting into the active-site tunnel formed by two lobes, while the acyl chain turns nearly $90^{\circ}$ and extends into the N-lobe as the R148A mutation opens up a pocket between the a5 helix (where the R148 residue is located) and the $\beta$ sheet (Fig. 6d). Interestingly, the $\varepsilon$ nitrogen of $\mathrm{H} 140$ (the second histidine of the 
a

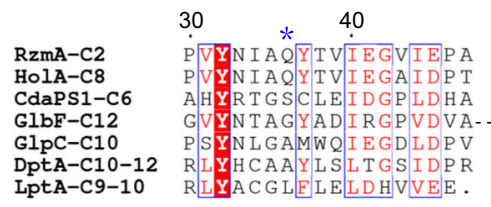

c

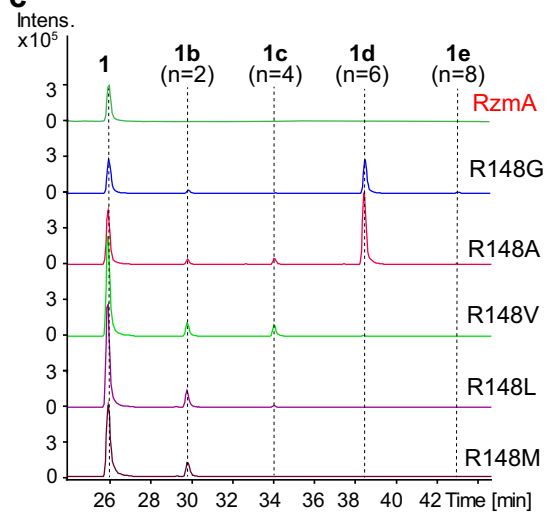

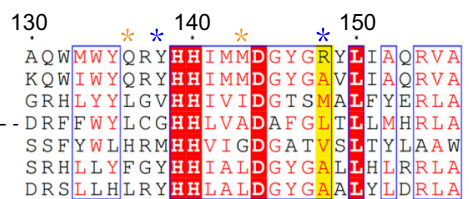

DRSLLHLRYHHIALDGYGA L I I I R R R A

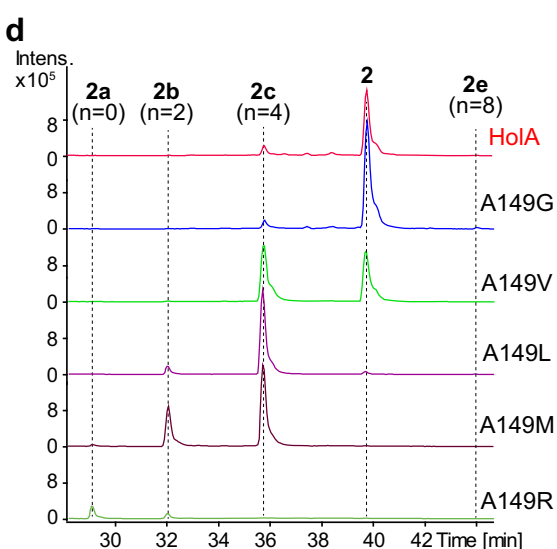

b
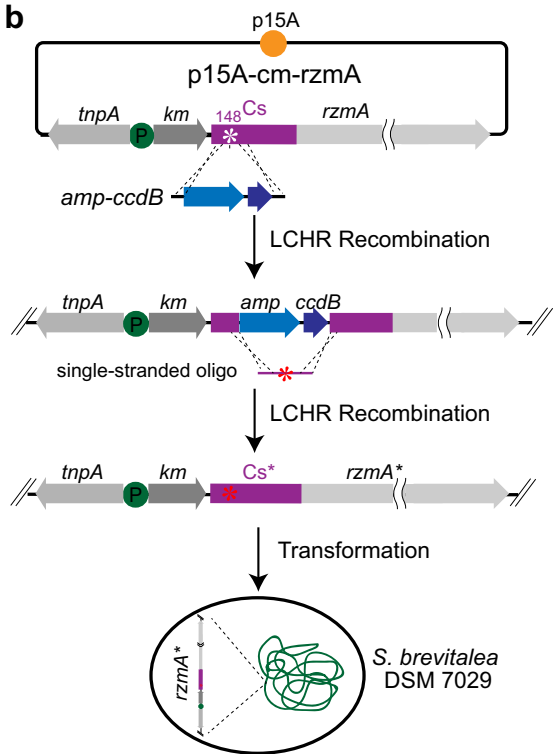

Fig. 4 Point mutations of a key residue in the RzmA-Cs and HolA-Cs domains. a Truncated sequence alignment of RzmA-Cs and HolA-Cs domains with other Cs domains loading different acyls. The asterisk-labeled residues represent key sites mentioned in the text for acyl substrate specificity (for details see Supplementary Fig. 4a). b The in vivo point mutation strategy of the rzmA-containing plasmid using CcdB counterselection recombineering 50 , 51 The point mutations in holA were also performed in the same way. c, d Comparative production analysis of point mutations of RzmA R148 (c) and HolA A149 (d) to different residues in vivo showed gradient changes of the acyl chains of rhizomide and holrhizin, respectively. EICs at $\mathrm{m} / \mathrm{z}=732.391$ (1), 760.421 (1b), 788.452 (1c), 816.483 (1d), and 844.513 (1e) for (c), and at $m / z=733.412$ (2a), 761.441 (2b), 789.472 (2c), 817.503 (2), and 845.537 (2e) for (d). Wild-type RzmA and HolA expressed in S. brevitalea DSM 7029 are labeled in red. LCHR linear plus circular homologous recombination, amp-ccdB cassette of ampicillin resistance gene and CcdB toxin genes; tnpA and km are same as in Fig. 2. Full information was given in "Methods" section and Supplementary files.

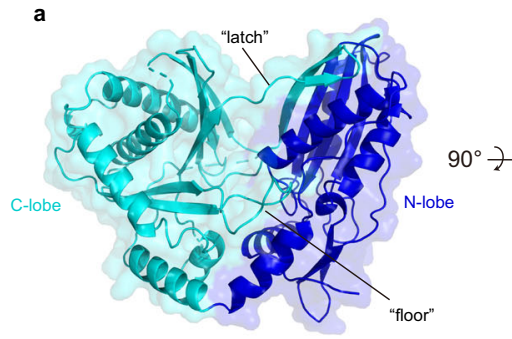

b

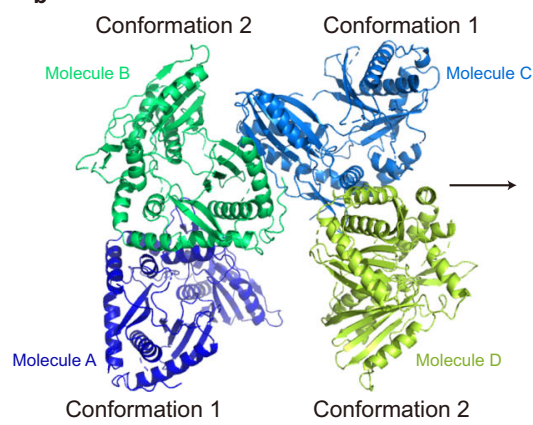

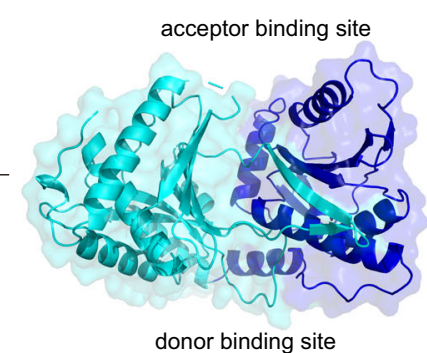

donor binding site
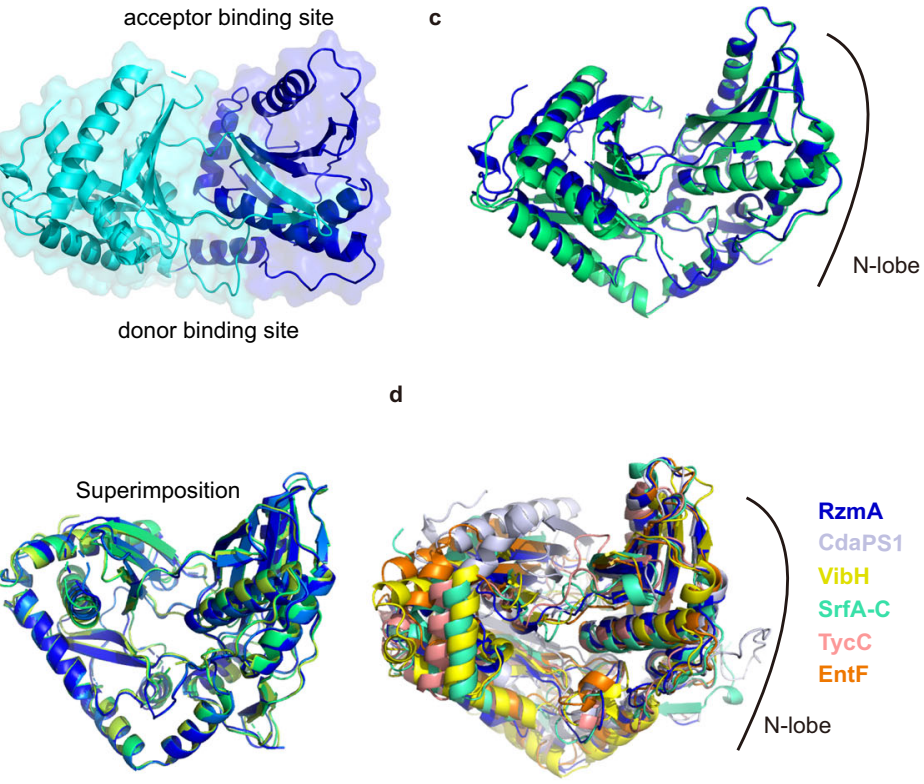

Fig. 5 The overall structure of the RzmA-Cs domain. a RzmA-Cs consists of N-lobe (blue) and C-lobe (cycan), and the C-lobe has two regions expanding to N-lobe, named as "latch" (residues 349-369) and "floor" (residues 274-287)39. b Four molecules of RzmA-Cs in each asymmetric unit with two conformations. c Comparison of two conformations of RzmA-Cs by overlaying the N-lobe. $\mathbf{d}$ Superimposition of $C$ domains in six NRPSs, RzmA (PDB: 7C1H), CdaPS1 (PDB: 4JN5), VibH (PDB: 1L5A), SrfA-C (PDB: 2VSQ), TycC (PDB: 2JGP), and EntF (PDB: 5JA1), by their N-lobes.

"HHxxxDG" motif) is relatively close to the acyl group of the donor substrate $(3.6 \AA)$, suggesting that the substrate positioning function of this histidine applies not only to the acceptor substrate as inferred previously ${ }^{19}$ but also to the donor substrate (the C8-CoA in this case). In addition, this structure helps us understand the conservation of G145 (within the "HHxxxDG" motif), because at this position in the $\mathrm{C}$ domain (starting point of the $a 5$ helix), the residue has to sacrifice its side-chain to make room for proper binding and positioning of the donor substrates (Fig. 6d). 
a

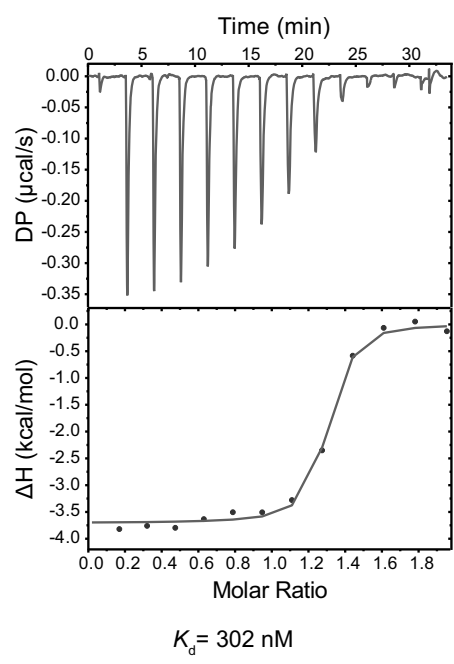

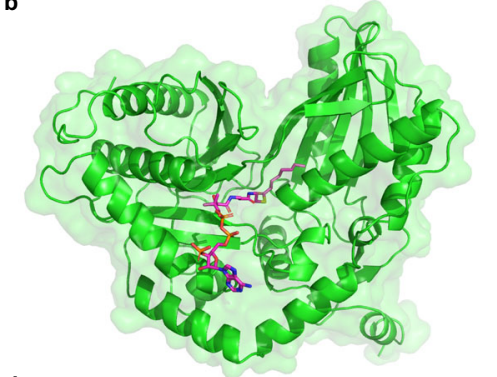

d

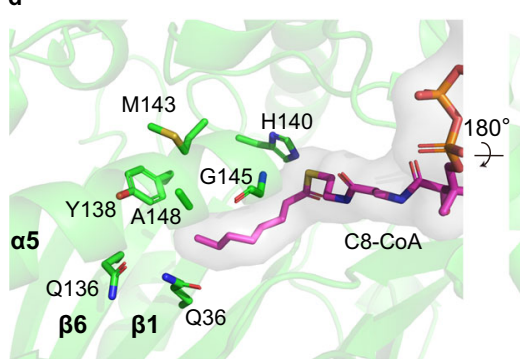

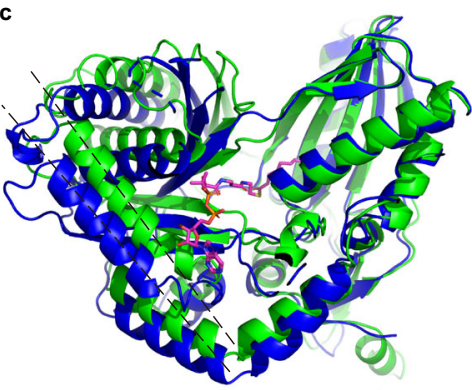

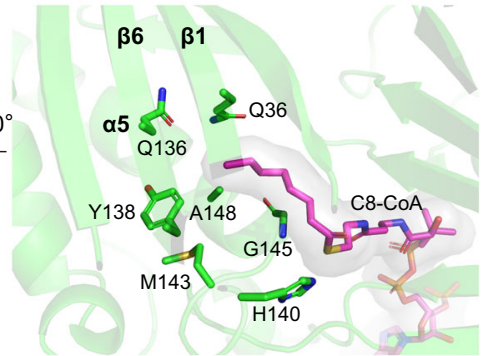

Fig. 6 The co-complex structure of RzmA-Cs R148A with C8-CoA. a Binding affinity measurement of C8-CoA to RzmA-Cs R148A using ITC. b Overall cocomplex structure of R148A with C8-CoA. c Superimposition of R148A in complex with C8-CoA (green) and R148A unliganded (blue) by their N-lobes, highlighting the conformational changes of $\mathrm{Cs}$ domains during substrate binding. $\mathbf{d}$ The enlarged views of $\mathrm{C} 8$-CoA binding site. All the residues in sticks mode show their side chains only, except for the G145 which does not have a side-chain.

As mutation of the pocket "entry gate" residue R148 to glycine resulted in C8-rhizomide (1d) as the main product together with a tiny portion of C10-rhizomide (1e) (Figs. 4a, c and 7a), this mutation was chosen for further combinational mutations to explore the possibility of extending the length of the acyl chain on rhizomide. We first checked the residues near the tip of the $\mathrm{C} 8$ chain in the structure for mutation candidates to expand the binding pocket (Fig. 6d). Four residues, Q36 on the $\beta 1$ strand, Q136 and Y138 on the $\beta 6$ strand, and M143 on the loop between $\beta 6$ and $\alpha 5$, were selected for this purpose. Mutations of these residues to Ala in combination with R148G clearly increased the length of the acyl chain incorporated into the peptidyl backbone both in vivo and in vitro (Fig. 7a, b). Compared with the mutation R148G alone, the additional mutations Q136A and M143A retained similar specificity for donor substrates, suggesting that Q136 and M143 may not significantly affect the length of the acyl chain. On the other hand, the additional mutations Y138A and Q36G changed the substrate preferences to longer acyl chains (C10-C12). However, the Q36G mutation markedly decreased the yields of rhizomide derivatives in vivo; thus, Y138 could be a superior mutation site for extension of acyl chains. The combined mutations Q136A/Y138A/R148G, Y138A/ M143A/R148G, and Q136A/Y138A/M143A/R148G further shifted the preferences to C12-C16 fatty acyl-CoAs (Fig. 7a, b). The structures of rhizomide derivatives (1b-1h) containing different acyls were identified by UPLC-HRMS/MS and NMR analysis (Supplementary Fig. 2, Supplementary Tables 1 and 2, Supplementary Figs. 21-28), confirming substrate alteration in vivo. The triple mutant Y138A/M143A/R148G showed a large change in the specificity of the RzmA-Cs domain for acyl chains from short C2 to long C14, up to C16, with no marked reduction in product yields in vitro and in vivo (Fig. 7a, b), thus probably providing the optimal mutation sites for the extension of acyl chains in lipopeptides. These results correlate well with our hypothesis that rational engineering by mutation of Cs residues to reshape the substrate-binding pocket of acyl chains can lead to the production of lipopeptides with modified or even desired acyl chains.
The mechanism of the lipoinitiation reaction. To elucidate the lipoinitiation mechanism, we aimed to obtain the co-complex structure of RzmA-Cs with both donor (acyl-CoA) and acceptor (amino acid) substrates. To simplify the crystallization process, we utilized the substrate mimic L-Leu-SNAC 32 as in the above in vitro assays instead of the real substrate L-Leu loaded on the PCP domain from the same initiation module of RzmA (Fig. 1a). However, our initial crystallization attempts with WT or R148A mutant proteins all failed, possibly due to the fast reaction of the two substrates. To diminish the reaction rate, we made an additional mutation on the second histidine of the "HHxxxDG" motif ${ }^{37}$ of RzmA-Cs R148A to create the double mutant H140V/ R148A, which finally enabled us to obtain several crystals in the absence or presence of substrates (Supplementary Table 5).

We then solved and analyzed these H140V/R148A structures. The overall structure of unbound H140V/R148A is almost identical to the structures of WT and R148A in both conformations (Supplementary Fig. 6a). Then, we compared the C8-CoAbound structures of H140V/R148A and R148A. Although their overall structures are quite similar (Supplementary Fig. 6b), we noticed that the electron densities surrounding the $\mathrm{C} 8$ acyl chain (but not the CoA portion) are much worse in the H140V/R148A structure than in the R148A structure (Supplementary Fig. 6c, d). These results indicate that the acyl chain of the substrate in the H140V/R148A structure is more flexible with H140 mutated to valine, again confirming the role of $\mathrm{H} 140$ in positioning the "donor" acyl-CoAs.

Next, we analyzed the structure of H140V/R148A in cocomplex with two substrates. Its overall structure is almost identical to that of H140V/R148A bound to only C8-CoA (Fig. 8a, Supplementary Fig. 7a), despite a small conformational difference in $\mathrm{C} 8$-CoA itself (Supplementary Fig. 7b). In addition, the electron densities around both C8-CoA and L-Leu-SNAC are clearly visible (Supplementary Fig. 7c), making us confidently pinpoint how the Cs residues within the active site interact with both substrates. In particular, by mutating the key residue H140 back from V140 in the structure, we were able to show that its side-chain points right in the middle of two substrates, with a 


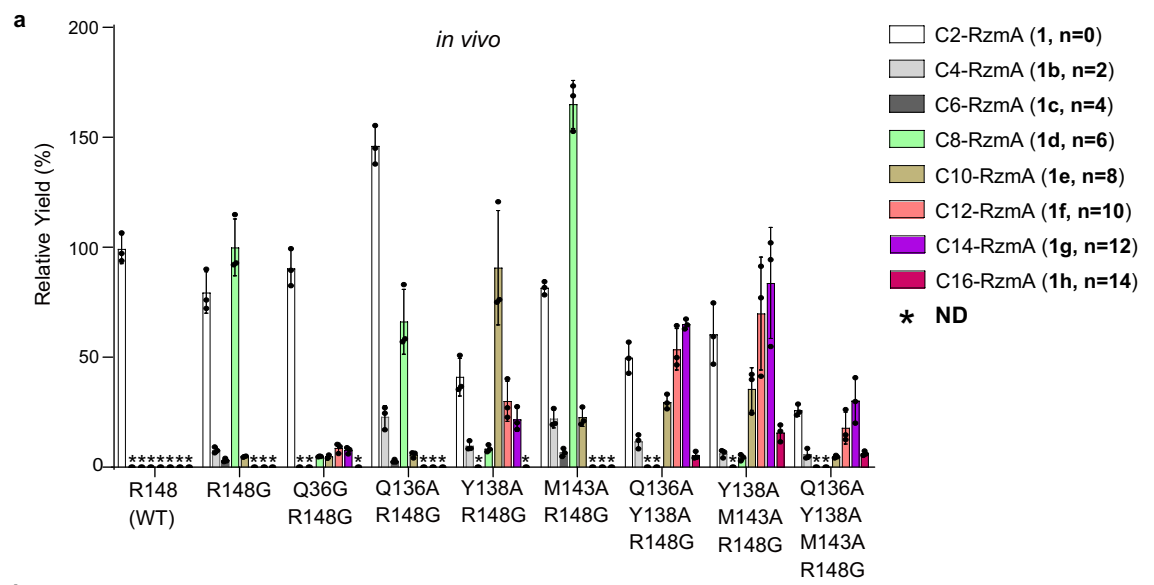

b

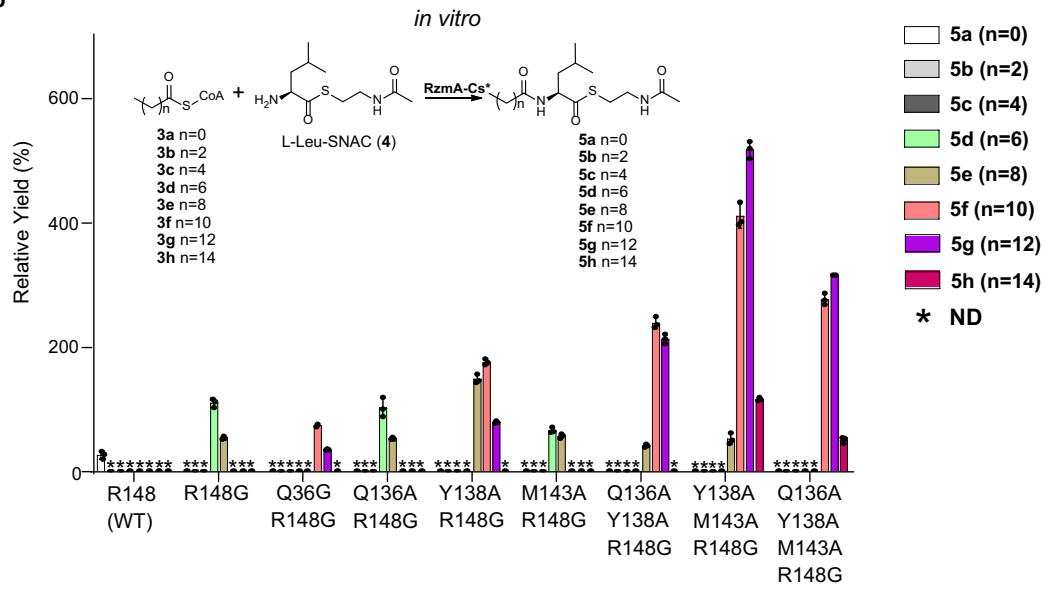

Fig. 7 Structure-guided in-depth engineering of the Cs domain of rhizomide. a The relative yields of rhizomide derivatives (1, 1b-1h) with different acyl chains (C2-C16) in S. brevitalea DSM 7029 harboring the corresponding point-mutated rzmA. The yield of 1d (C8-RzmA) in RzmA-R148G was quantified as a reference $(100 \%)$ for the peak areas of EICs. Related chemical structures of rhizomide derivatives were given in Fig. $1 \mathrm{~b}$ in which the ' $n$ ' represents the number of $\mathrm{CH}_{2}$ group. $\mathbf{b}$ The relative yields of acyl-Leu-SNAC (5a-5h) in the in vitro acyl-CoAs substrate competition experiment of RzmA-Cs variants. The yield of $\mathbf{5 d}$ (C8-Leu-SNAC) in RzmA-Cs R148G was quantified as a reference (100\%) for the peak areas of ElCs. The products that were not detected (ND) by UPLC-MS are marked with asterisks. Experiments were conducted in triplicates $(n=3)$, data are presented as mean values $+/-$ SD. The source data is provided in the Source data file.

distance of $<4 \AA$ to the reactive atoms from both sides (i.e., the "donor" carbonyl group and the "acceptor" $\alpha$-amino group as shown in Fig. 8b). This structure, along with other structures described above or previously ${ }^{19}$, undoubtedly indicates the key function of the second histidine at the "HHxxxDG" motif of Cs domains in positioning both substrates.

Finally, to depict how RzmA-Cs recognizes amino acids on the "acceptor" side, we zoomed into the binding site of L-Leu-SNAC in our structure (Supplementary Fig. 7d), and compared it with that of the chemical probe (as a substrate mimic) covalently bound to the Cs domain of the CdaPS1 E17C mutant (Fig. 8c) ${ }^{19}$. In the RzmA-Cs structure, three residues, P271, S287, and T289 (corresponding to P292, S309, and R311 in CdaPS1) near the side-chain of L-Leu may participate in substrate recognition (Fig. 8c). Interestingly, these three RzmA residues are fully conserved in HolA (Supplementary Fig. 4a), whose Cs domain accepts L-Val instead of L-Leu in the holrhizin biosynthetic pathway (Fig. 1c). Therefore, it was not surprising that RzmA-Cs could incorporate L-Val as the substrate both in vivo (Fig. 2c, d) and in vitro (Fig. $3 c, d$ ). In addition, the sequence alignment of several other Cs domains with the same or different substrates revealed no strict rule of conservation for the above three residues, except that the vast majority of them are short-chain residues (Supplementary Fig. 4a). The above in vitro experiments with the RzmA-Cs, HolA-Cs, and GlbF-Cs domains also showed relatively wide specificities for acceptor substrates (Fig. 3c, d). All the above results are consistent with the fact that Cs domains can accommodate a certain degree of variation in the "acceptor" substrates, while the neighboring A domains are more responsible for substrate selectivity. This finding also supports the Cs domain swapping strategy to change the acyl chains (Figs. 2c, d, 3a, b), and the NRPS module swapping strategy at the $\mathrm{C}-\mathrm{A}$ linker region for the combinatorial biosynthesis of nonribosomal peptides ${ }^{36,38}$.

Conformational changes in the reaction cycle. In addition to the above three structures of the RzmA-Cs H140V/R148A mutant in either unbound or substrate-bound forms, we obtained a fourth ligand-free structure (Supplementary Table 5) derived from crystals that grew in drops containing H140V/R148A proteins and two substrates. However, these crystals usually appeared with a unique morphology after 2 weeks, much later than those in the co-complex with both substrates, which grew under the same conditions but took only 1-2 days (Supplementary Fig. 8). Interestingly, unlike the other unbound structures (WT, R148A, or H140V/R148A), which showed two conformations in the $P 1$ space group, this structure of $\mathrm{H} 140 \mathrm{~V} / \mathrm{R} 148 \mathrm{~A}$ was solved with only one conformation in the $P 2_{1} 2_{1} 2_{1}$ space group (Fig. 9a). 
a
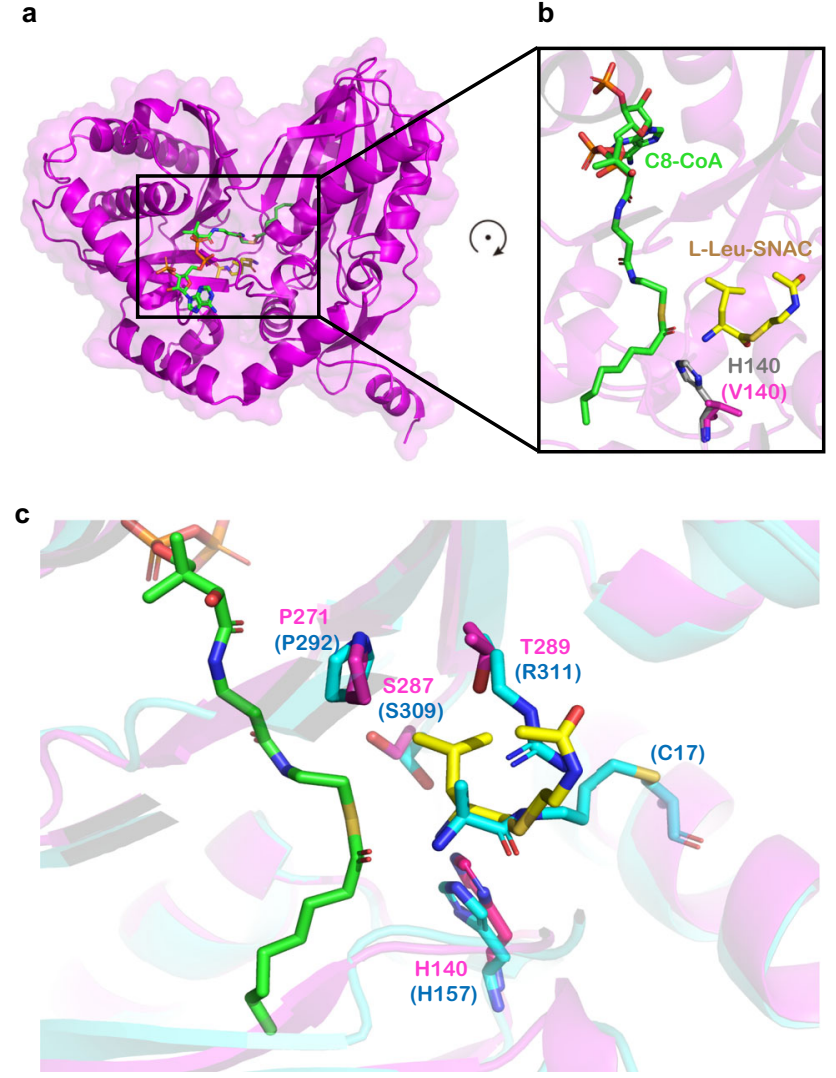

Fig. 8 Interactions between RzmA-Cs and both substrates. a Overall structure of the H140V/R148A mutant of RzmA-Cs in co-complex with C8CoA (green) and L-Leu-SNAC (yellow). b An enlarged view around the two substrates. Valine 140 (magenta) is mutated back to histidine (white) to show its spatial position relative to the substrates. c Comparison of the L-Leu-SNAC binding site in RzmA-Cs with that of the covalent substrate mimic in the CdaPS1-Cs E17C mutant (PDB code 5DU9). Residues near the "acceptor" substrates in RzmA and CdaPS1 are labeled in magenta and blue, respectively.

Moreover, when comparing the structure of this ligand-free H140V/R148A with those of other substrate-unbound or substrate-bound forms (Supplementary Fig. 9a, b), we found that its overall conformation is very similar to that of substrate-bound H140V/R148A, with the majority of differences lying in the socalled "latch" and "floor" regions (Fig. 9b) ${ }^{39}$. This comparison indicated that the overturn of these two regions (especially the "floor" loop) dramatically increased the size of the active-site tunnel and expelled the C8-CoA substrate from the Cs domain (Supplementary Fig. 9c).

These above findings all point to the conclusion that we probably captured a "product-released" form of RzmA-Cs $\mathrm{H} 140 \mathrm{~V} / \mathrm{R} 148 \mathrm{~A}$. If this idea is correct, we should be able to detect the released product catalyzed by H140V/R148A in vitro. Therefore, we set up a testing system with a longer reaction time, and indeed detected the condensation product 5d after a 300-min incubation of $\mathrm{H} 140 \mathrm{~V} / \mathrm{R} 148 \mathrm{~A}$ at $30^{\circ} \mathrm{C}$, but in an extremely low yield compared with that of R148A after 100-min incubation (Fig. 9c). Thus, the H140V mutation did not completely abolish the condensation activity, correlating well with its role in substrate positioning ${ }^{12,19}$. Based on these H140V/R148A structures and related functional assays, we propose three potential conformational states (i.e., "unbound-bound-released") within the lipoinitiation reaction cycle catalyzed by Cs domains (Fig. 9d, also illustrated in Supplementary Video 1). The major conformational changes during this cycle reflect the relative positions of two lobes, in concert with the binding of substrates and release of products. Notably, the "latch" and "floor" regions play essential roles in mediating and may also responding to conformational changes, as they are both crossover structures stretching from the C-lobe to the $\mathrm{N}$-lobe, and their positions together determine the shape of the active-site tunnel (Supplementary Fig. 9c). Upon substrate binding, the two lobes of the Cs domain may close up to form proper interactions with the substrates, while a number of residues in the "latch" and "floor" regions may flip their side chains toward the active site to shrink the tunnel. After the condensation reaction, the Cs domain may open up slightly, accompanied by dramatic movement of the "floor" loop up against the binding position of CoA, possibly forcing it out of the "donor" site. Finally, the two lobes may open more, and the "floor" loop may move back down, from the "released" to the "unbound" position for a new cycle. This reaction cycle could also apply to other $\mathrm{C}$ domains, since their structures and functions are both closely related to those of Cs domains.

\section{Discussion}

The lipopeptide natural products generated by the NRPSs containing Cs domains exhibit a wide range of bioactivities. These include antibiotics, anticancer drug candidates, and biosurfactants ${ }^{4-7}$. Some bacterial lipopeptides play roles in antagonism toward other (micro)organisms, motility, and attachment to surfaces ${ }^{9}$. Their biological activity in general depends on the interaction of their amphipathic structure with the cell membranes of target organisms ${ }^{40}$. Changing the acyl chain can balance the bioactivity and toxicity of lipopeptides, as shown in the development of daptomycin ${ }^{4,15}$. Several strategies have been harnessed to generate lipopeptide derivatives with different lipid groups for bioactivity evaluation. The classical semisynthetic method involves the deacylation of the native acyl chain by acylases to generate the peptide nucleus and subsequent reacylation with a desired acyl group by chemical reaction, which requires burdensome screening of efficient acylases and cumbersome (de)protection of other polar groups ${ }^{15,16}$. The genetic engineering method to change the acyl chain often relies on supplementing with alternate donor substrates based on manipulation of the lipid biosynthetic pathway or mutasynthesis. Directed mutagenesis of the active site of the $\beta$-ketoacyl-ACP synthase FabF3 of Streptomyces coelicolor, an enzyme in the biosynthesis of the CDA fatty acid moiety (C6), led to successful generation of two novel CDA derivatives with truncated (C4) acyl side chains ${ }^{41}$. Disruption of the biosynthetic gene for the native acyl chain of pneumocandin combined with the feeding of alternative side-chain precursors yielded four new pneumocandin congeners with straight C14-C16 side chains ${ }^{42}$. However, the yields were low, as the tolerance of the Cs domain for donor and acceptor substrates is limited.

Here, we changed the acyl chains of nonribosomal lipopeptides by bioengineering Cs domain in two ways, Cs domain swapping and point mutation. The recently identified exchange units (XUs) assembled at the fusion point located in the $\mathrm{C}-\mathrm{A}$ linker region and the exchange unit condensation domain (XUC) fused at the point between the $\mathrm{N}$-terminal and $\mathrm{C}$-terminal subdomain of the $\mathrm{C}$ domain enabled the production of novel nonribosomal peptides without a significant decrease in yield ${ }^{38,43}$. We exchanged full-length Cs domains including the Cs-A linker or N-terminal subdomains (N-lobe), to alter the acyl chain length of lipopeptides. The full-length Cs domain exchange provided higher product yields than the $\mathrm{N}$-terminal subdomain swapping method. The relatively broad specificities of the Cs domain for acceptor 
a

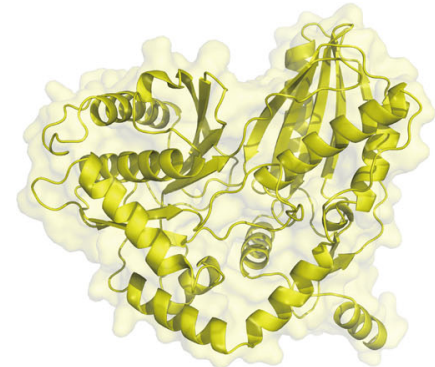

c

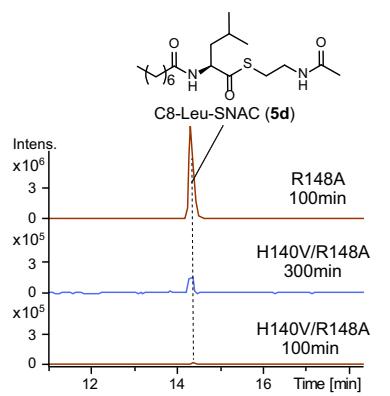

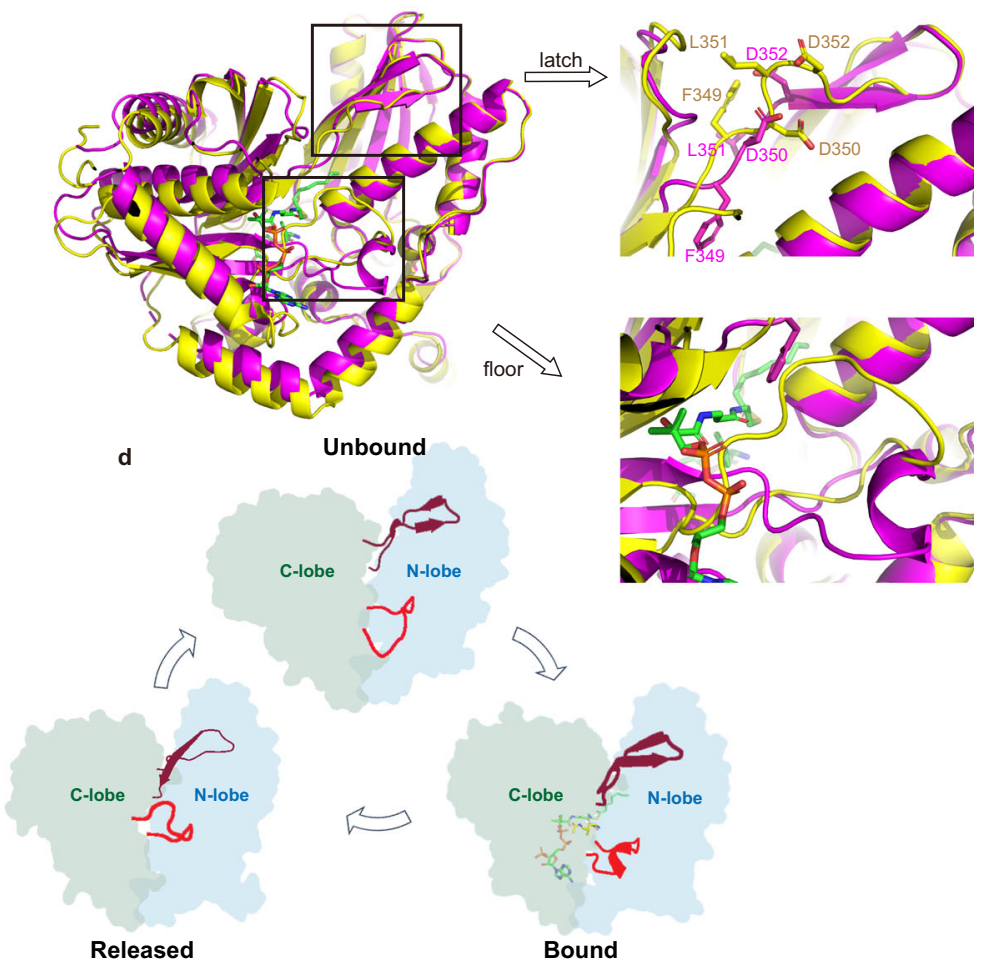

Fig. 9 Potential conformational changes within the reaction cycle of lipoinitiation. a The overall structure of RzmA-Cs H140V/R148A in a "productreleased" state. b Superimposition of H140V/R148A structures in "released" (yellow) and "bound" (magenta) forms. Two enlarged views highlighting the changes in the "latch" and "floor" regions. c The comparative analysis of C8-Leu-SNAC (5d) in vitro produced by RzmA-Cs mutants R148A and H140V/ R148A with substrates C8-CoA and L-Leu-SNAC. EICs at $m / z=359.2\left(\mathbf{5 d},[\mathrm{M}+\mathrm{H}]^{+}\right)$. d A schematic diagram showing the potential conformational changes of Cs domains during the reaction cycle, with the "latch" and "floor" regions (secondary structures in cartoon mode) highlighted in brown and red, respectively.

substrates shown in vitro (Fig. 3c, d) support full-length Cs domain swapping, but the specificity of the contiguous A domain should also be considered to maximize the production of engineered products with different acyls. The suitable recombination sites between the Cs and A domains need to be further investigated in detail for the efficient engineering of lipopeptides. The recently reported research on the modification of NRPSs by A domain alone substitution also supported that $\mathrm{C}$ domain specificities were not as strict as we previously thought ${ }^{36}$. Our results suggested that the use of full-length Cs domain as a swapping unit is a feasible way to modify lipopeptides.

On the basis of the complex structure of the Cs domain with acyl-CoA and of both in vitro and in vivo mutation experiments in this investigation, we revealed the binding pocket of acyl group and the key amino acid residues that control the length of the acyl group. A recent study using modeling and in vitro experiments identified four residues as functionally related to the fatty acyl substrate selectivity of LptA-C $1^{12}$, but the residues were different from our revealed key sites demonstrated by the cocrystal structure and by in vitro and in vivo experiments. We found that three sites in RzmA-Cs Q36, Y138, and R148 play key roles in controlling the specificity of the acyl chain, in contrast to the previous study on LptA-C1 in A54145 biosynthetic pathway ${ }^{12}$, i.e., A152, A369, A386, and L397 (corresponding to Y149, D350, N367, and Y378 in RzmA-Cs) (Supplementary Fig. 4a). We also noticed the inconsistencies between our in vivo and in vitro experiments, due to the complexity of the in vivo system and/or to different products. The in vitro experiments determined only the formation of the first simple biosynthetic intermediate mimics by a Cs domain-catalyzed reaction, while the in vivo experiments showed the final complex products after many reactions. The concentrations of the acyl-CoA substrates vary widely in cells; e.g., acetyl-CoA, as an important primary metabolite, is present at concentrations at least one or two orders of magnitude higher than those of other medium or long fatty acyl-CoAs in E. colit4. We set up another in vitro experiment to simulate in vivo conditions using higher concentrations of acetyl-CoA and butanoylCoA (C2-CoA:C4-CoA:C6 to C18-CoA = 1000:100:1) and an extended reaction time, and the products C2-Leu-SNAC and C4Leu-SNAC were detected with relatively high yields, as shown by the counterparts in the in vivo experiments (Supplementary Fig. 10). This result signified that substrate concentration and reaction time also influenced the in vitro reaction, suggesting that the donor specificity of the Cs domain was not very strict, although the optimal products could be obtained. Triple mutations in the RzmA-Cs domain (Y138A/M143A/R148G) can greatly extend the acyl chain of rhizomide A from C2 to C16 in vivo and in vitro, which proved that point mutations in the Cs domain can change the acyl chains of lipopeptides. Thus, we may establish a foundation to artificially generate lipopeptides with varying lengths of acyl chains for biological evaluation. However, more combinatorial mutations of the key sites in the acyl-CoA building pocket are required to achieve the precise control of acyl chain length. This Cs domain bioengineering strategy including domain swapping and point mutation, is a complement to attempts to modify the peptidyl backbone in the field of combinatorial biosynthesis to produce novel lipopeptides and nonribosomal peptides.

The co-complex structures of an enzyme and its substrates or intermediate analogs usually provide direct evidence for the 


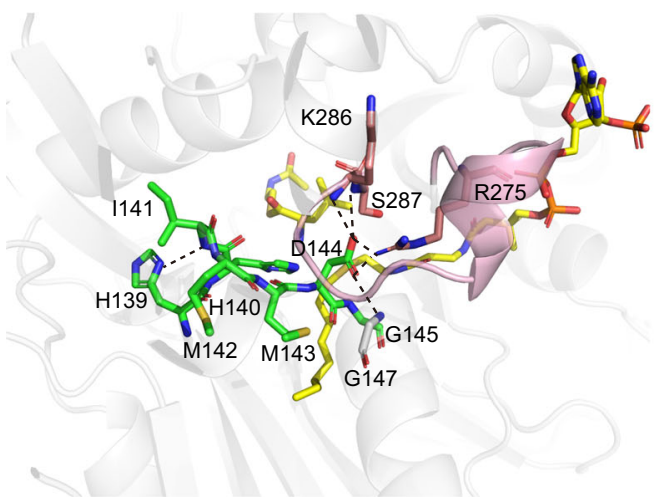

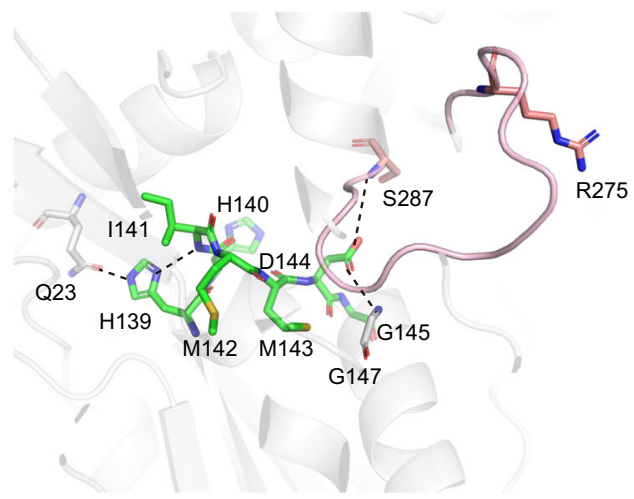

Fig. 10 Potential structure-stabilizing function of the conserved "HHxxxDG" motif. a As revealed by the co-complex structure of RzmA-Cs with two substrates, the side-chain of H139 interacts with the main chain of I141, and that of D144 interacts with the side-chain of R275 (to form a salt bridge) and the main chain atoms of G147, K286, and S287. b In the "unbound" structure of RzmA-Cs, most of the above residues interacting with H139 and D144 are at the similar positions, while the R275 residue of the "floor" region is far away from D144. The seven residues in the conserved "HHxxxDG" motif are colored in green, and the "floor" region is highlighted in light pink. The two bound substrates are colored yellow. All interactions are shown as black dashed lines.

catalytic mechanism. For the NRPS Cs domains, how the conserved "HHxxxDG" motif participates in condensation reactions is an interesting question that is not yet fully answered. A previous study on the CdaPS1-Cs domain suggested that the "catalytic histidine" (second histidine within the motif) is responsible for positioning the $\alpha$-amino group of acceptor substrates ${ }^{19}$. Our co-complex structures of RzmA-Cs not only supported this conception, but also extended the positioning function of this histidine $(\mathrm{H} 140)$ to the donor substrates, i.e., acyl-CoAs. In addition, we infer that the glycine within the motif (G145) is conserved as a residue without a side-chain to make room for the donor substrates. The other two conserved residues of the motif (H139 and D144 in RzmA-Cs), may help maintain the appropriate architecture around the active site, since their side chains are involved in multiple interactions with neighboring residues (Fig. 10a). It is noteworthy that among these D144-interacting resides, R275 undergoes a very large conformational change from the "unbound" status to contact D144 (Fig. 10b), as does the "floor" region of RzmA-Cs (see below). This salt bridge between D144 and R275 is potentially critical for the proper structure and function of Cs domains, as both residues are highly conserved in the sequence alignment (Supplementary Fig. 4a).

NRPSs are megaenzymes that conduct peptide biosynthesis like an assembly line, which requires cooperative conformational changes in all domains and modules. Recent structural studies on multidomain NRPS proteins visualized the conformational flexibility among different domains even modules during the biosynthetic reaction ${ }^{21-24,27}$. However, other than the various degrees of openness between the two lobes of the $\mathrm{C}$ domains ${ }^{39}$, the conformational changes in this domain during the condensation process have not been fully revealed, due to the lack of co-complex structures with substrates. Fortunately, we were able to capture several snap-shots of the conformations of RzmA-Cs, illustrating a potential reaction cycle including the "unboundbound-released" states (Supplementary Video 1). The most dramatic changes occurred in the "latch" and "floor" regions, which together define the shape and size of the active-site tunnel. In particular, the position of the "floor" loop in the "released" state overlapped with the binding position of CoA in RzmA-Cs, suggesting that this loop may help push the CoA out of the donor binding site after condensation (Fig. 9b). This function of the "floor" loop echoes previous findings that its counterpart loop in the epimerization domain of gramicidin $S$ synthetase (GrsA), interacts directly with the neighboring PCP domain at the donor site $^{45}$. Further investigations are much warranted to determine how the conformational changes of $\mathrm{C}$ domains function in concert with larger domain movements within a module or even across modules of NRPSs.

Collectively, here, our genetic engineering and cocrystal structures revealed how Cs domains recognize and interact with both donor and acceptor substrates, and we observed their unique conformational changes during the reaction cycle. Our work provides further insights into the mechanism of lipoinitiation, and suggests a feasible approach to generate lipopeptides with various acyl chains, facilitating future bioengineering efforts to produce more novel nonribosomal peptides.

\section{Methods}

General methods. All bacteria strains, plasmids, and sequences of primers used in this study are supplied in Supplementary Data 1. Detailed description for the construction of plasmids and related strains/plasmids/mutants are also given in Supplementary Data 1.

The Paraburkholderia rhizoxinica HKI 454 (DSM 19002), Schlegelella brevitalea DSM 7029 and its mutants were cultured in CYMG (8g/l Casein peptone, $4 \mathrm{~g} / \mathrm{l}$ Yeast extract, $4.06 \mathrm{~g} / \mathrm{l} \mathrm{MgCl} \cdot 2 \mathrm{H}_{2} \mathrm{O}, 10 \mathrm{ml} / \mathrm{l}$ glycerin) broth or agar plates with apramycin $(20 \mu \mathrm{g} / \mathrm{ml})$, kanamycin $(20 \mu \mathrm{g} / \mathrm{ml})$, gentamicin $(15 \mu \mathrm{g} / \mathrm{ml})$,

or chloramphenicol $(15 \mu \mathrm{g} / \mathrm{ml})$ as required. The $E$. coli cells for recombineering and plasmid propagation were cultured in Luria-Bertani (LB) broth or on LB-agar plates $(1.2 \%$ agar) with ampicillin $(100 \mu \mathrm{g} / \mathrm{ml})$, kanamycin $(15 \mu \mathrm{g} / \mathrm{ml})$, chloramphenicol $(15 \mu \mathrm{g} / \mathrm{ml})$, or gentamicin $(5 \mu \mathrm{g} / \mathrm{ml})$ as required. Reagents were purchased from New England Biolabs, Thermo Fisher Scientific, Invitrogen, and Sigma-Aldrich. DNA primers were purchased from Tsingke Biological Technology and Sangon Biotech.

Cloning and heterologous expression of rhizomide and holrhizin biosynthetic gene clusters. The rhizomide and holrhizin gene clusters, rzmA (RBRH_RS12370) and holA (RBRH_RS16800), were directly cloned from the genomic DNA (Genbank: NC_014718.1) of P. rhizoxinica HKI 454 using Red/ET recombineering in E. coli, respectively. The direct cloning experiments were performed according to the previous protocols ${ }^{46-48}$. In brief, genomic DNA is digested by appropriated restriction enzymes and purified by ethanol precipitation. The digested genomic DNA, p15A vector with homology, T4 polymerase, and buffer are mixed and reacted in a thermocycler in program: $25^{\circ} \mathrm{C}, 60 \mathrm{~min} ; 75^{\circ} \mathrm{C}, 20 \mathrm{~min} ; 50^{\circ} \mathrm{C}, 60 \mathrm{~min}$. The mixture is then electroporated into the L-arabinose induced E. coli GB05-dir (Supplementary Data $1-$ Bacteria strains) competent cells and plated on appropriated LB-agar plates to incubate at $37^{\circ} \mathrm{C}$ overnight. The $r z m A(23.8 \mathrm{~kb})$ and holA $(22.3 \mathrm{~kb})$ regions were cloned from digested genome mixture (ScaI and SalI for $r z m A$, EcoRV for holA) into the corresponding p15A-cm vectors in E. coli, and the resulting plasmids p15A-cm-rzmA and p15A-cm-holA were then inserted transferring IR-oriT-tnpA-IR- $k m$ cassettes to get final expression plasmids p15A-tnpAkm-rzmA and p15A-tnpA-km-holA, respectively. The rzmA and holA gene clusters 
are under the control of $\mathrm{P}_{\mathrm{Tn} 5-\mathrm{km}}$ constitutive promoter ${ }^{33}$. The primers for direct cloning were listed in Supplementary Data 1.

Then, both plasmids were electroporated into the heterologous host Schlegelella brevitalea DSM 7029 (previous known as Burkholderiales strain DSM 7029 or Polyangium brachysporum K481-B101 ${ }^{33,34,49}$ for functional expression according to the previous method 29,33 . The correct colonies were checked by colony PCR. The primers were listed in Supplementary Data 1. The verified mutants containing $r z m A$ or holA were cultured overnight and inoculated into $50 \mathrm{ml}$ antibiotic supplemented CYMG medium in a $250 \mathrm{ml}$ flask and incubated at $30^{\circ} \mathrm{C}, 200 \mathrm{rpm}$ for 4 days. The resin Amberlite XAD-16 (2\%) was added into the culture and continually incubated for another day. The cells and resins were collected by centrifugation and extracted with $40 \mathrm{ml} \mathrm{MeOH}$ for $2 \mathrm{~h}$. The supernatant was concentrated in vacuo and the resulting residue was dissolved in $1 \mathrm{ml} \mathrm{MeOH}$ for further metabolic analysis by UPLC-MS or UPLC-HRMS.

Cs (sub)domain swapping. The Cs domain swapping was also conducted by LLHR of recombineering ${ }^{46}$. The procedure was as same as did in direct cloning experiments by using linear DNA and digested vectors. For $r z m A$ gene cluster, a linear fragment harboring HolA-Cs domain (NCBI: WP_041754829.1, residues 1-479) and $k m$-IR-tnpA-oriT-IR cassette flanked with homology arms were amplified from p15A-tnpA-km-holA by PCR. The p15A-cm-rzmA was linearized by digestion of ScaI and NheI. Two linear fragments were recombined to form plasmid p15AtnpA-km-rzmACsholA (RCH in Fig. 2a, c) in which the RzmA-Cs domain (NCBI: WP_013428324.1, residues 1-479) was replaced by HolA-Cs domain (Fig. 2a). Likewise, plasmid p15A-tnpA-km-holACsrzmA (HCR in Fig. 2d) was also constructed where the HolA-Cs was changed to RzmA-Cs domain, except the p15A$\mathrm{cm}$-holA was linearized by digestion of HindIII and EcoRV. The swapping of Cs subdomain (N-lobe of Cs domain) followed the same procedure as the full-length Cs swapping method by using fragments RzmA-CsN (residues 1-188) or HolACsN (residues 1-189) and corresponding original plasmids p15A-cm-rzmA and p15A-cm-holA were linearized by digestion of ScaI/BspQI and EcoRV (Fig. 2b-d), respectively. The resulting plasmids were verified and transformed into S. brevitalea DSM 7029 for heterologous expression and metabolic analysis. Detailed description for the construction of plasmids and related strains/mutants/primers were given in Supplementary Data 1.

For glidobactin gene cluster $(g l b)$, the Cs domain swapping was performed in the native producer S. brevitalea DSM 7029 using Reda $\beta 7029$ recombineering ${ }^{29}$. HolA-Cs and GlpC-Cs domains were used to replace GlbF-Cs domain. First, the GlpC-Cs encoding region in DSM 7029 was replaced by a gentamicin resistance gene to obtain mutant $7029 \Delta \mathrm{GP}$ avoid interference of homologous recombination. Then an apraphiC31 cassette was used to replace the tnpA in p15A-tnpA-km-holA or p15A-tnpA$\mathrm{km}$-rzmA to form the cassette, respectively. Then this cassette flanked with homology arms was amplified by PCR and it was to replace the GlbF-Cs domain in 7029 $\mathrm{GP}$. The resulting mutant $7029 \mathrm{H} \Delta \mathrm{GP}$ or $7029 \mathrm{R} \Delta \mathrm{GP}$ contains a chimeric $g l b F$ with HolACs or RzmA-Cs under the control of $\mathrm{P}_{\mathrm{Tn} 5-\mathrm{km}}$ promoter. The km-apra cassette was connected to GlpC-Cs encoding region by overlapping PCR, then it was used to replace the GlbF-Cs domain in 7029 $\Delta$ GP to get mutant 7029P $\Delta$ GP. N-terminal GlpCCs hybrid $g l b F$ was constructed in the same way by using the overlapped GlpC-CsN$k m$-apra cassette $(7029 \mathrm{PN} \Delta \mathrm{GP})$. All mutants were verified by colony PCR and sequencing. The correct mutants were cultured and analyzed as mentioned above. The $\mathrm{P}_{\mathrm{Tn} 5-\mathrm{km}}$ promotor was also inserted in front of $g l b F$ to make a control for metabolic analysis (7029B $\Delta \mathrm{GP})$. Detailed description for the construction of $a m p-c c d B$ plasmids and related strains/plasmids/mutants were given in Supplementary Data 1.

Point mutations of Cs domains in rzmA and holA gene clusters. The seamless point mutations in the large biosynthetic genes were created by $\mathrm{CcdB}$ counterselectionbased recombineering reported previously ${ }^{50,51}$. This method mentioned two rounds of recombineering: the site of interest was first replaced by a counterselection cassette $a m p-c c d B$ in $E$. coli GBred-gyrA462; then a synthetic singlestrand oligonucleotide containing a mutation of interest to replace this cassette under the counterselection of the toxicity marker inferred by the counter-selectable gene $c c d B$. All single mutation R148M, R148L, R148V, R148A, R148G of RzmA-Cs in rzmA (p15A-tnpA-km-rzmA and pET28a-rzmACs) and A149M, A149L, A149V, A149R, A149G of HolA-Cs in holA (p15A-tnpA-km-holA and pET28aholACs) were introduced using this method and verified by sequencing, respectively. The double or multiple mutants including Q36G/R148G (Q36G), Q136A/ R148G (Q136A), Y138A/R148G (Y138A), M143A/R148G (M143A), Q136A/ Y138A/R148G (QYA), Y138A/M143A/R148G (YMA), and Q136A/Y138A/ M143A/R148G (QYMA), H140V/R148A of RzmA-Cs in rzmA (p15A-tnpA-kmrzmA and pET28a-rzmACs) were also achieved using repeated counterselection recombineering. All primers used for point mutations listed in Supplementary Data 1. The correct plasmids containing biosynthetic gene clusters were transferred into S. brevitalea DSM 7029 for heterologous expression and metabolic analysis, respectively. The Cs domain variants were transferred into E. coli BL21 for protein expression and purification. Full information for the construction of plasmids and related strains/mutants/primers were given in Supplementary Data 1.

Metabolic analysis. The metabolic analysis of mutants was performed by UPLC-MS and UPLC-HRMS. The UPLC-MS was same as mentioned in our previous publication $^{52}$. The UPLC-HRMS analysis was same as described before except the UPLC system which was performed on a column (YMC, $\mathrm{C}_{18}, 250 \times 4.6 \mathrm{~mm}, 5 \mu \mathrm{m}$ ) with gradient elution at a constant flow rate of $1 \mathrm{ml} / \mathrm{min}, 0-5 \mathrm{~min}, 5 \% \mathrm{~B} ; 5-55 \mathrm{~min}$, $5-95 \% \mathrm{~B} ; 55-60 \mathrm{~min}, 95 \% \mathrm{~B}^{29}$. Mass spectra were acquired in $\mathrm{m} / \mathrm{z}$ in a positive ionization mode with auto MS2 fragmentations. All small-scale fermentations and analyses followed the same method as described above.

Purification of compounds. The derivatives of rhizomide A and holrhizin A were purified according to our previous description with minor modifications ${ }^{29,30}$. For compound 1d, the extract of $12 \mathrm{~L}$ culture of mutant 7029WT::p15A-tnpA-kmrzmACsholA (7029RCH, Fig. 2c) was eluted by a normal-phase silica gel column resulting in fraction of $\mathrm{CH}_{2} \mathrm{Cl}_{2}: \mathrm{MeOH}=15: 1$ which was further purified by semipreparative reversed-phase high-performance liquid chromatography (RPHPLC) (column: YMC-Pack ODS-A $\mathrm{C}_{18} ; 5 \mu \mathrm{m}, 250 \times 10 \mathrm{~mm}$; gradient elution: $0-5$ min, $65 \%$ ACN; 5-25 min, 65-68\% ACN; $25.1-35$ min, 95\% ACN) to afford 1d (6.4 mg, white amorphous solid) at retention time $21.5 \mathrm{~min}$. For compound $2 \mathrm{a}$, the extract of $12 \mathrm{~L}$ culture of mutant 7029WT::p15A-tnpA-km-holACsrzmA (7029HCR, Fig. 2d) was eluted by gradient elution resulting in fraction of $\mathrm{CH}_{2} \mathrm{Cl}_{2}$ : $\mathrm{MeOH}=10: 1$ which was further purified by semipreparative RP-HPLC (column: YMC-Pack ODS-A C $18 ; 5 \mu \mathrm{m}, 250 \times 10 \mathrm{~mm}$; gradient elution: $0-5 \mathrm{~min}, 30 \% \mathrm{ACN}$; 5-25 min, 30-45\% ACN; $25.1-35 \mathrm{~min}, 95 \% \mathrm{ACN}$ ) to obtain $2 \mathrm{a}$ (5 mg, white amorphous solid) at retention time $21.6 \mathrm{~min}$. The $\mathbf{1 d}$ and $\mathbf{2 a}$ were dissolved into DSMO- $d_{6}$ for NMR recording (Bruker Avance III-600 spectrometer) and the NMR data were summarized in Supplementary Tables 1-3 and the NMR spectra were shown in Supplementary Figs. 11-20, respectively. For compounds 1e-1h, the extract of $20 \mathrm{~L}$ culture of mutant 7029WT::p15A-tnpA-km-rzmA-R148G-Y138AM143A (7029YMA, Fig. 7a) was eluted by a normal-phase silica gel column. The elution of $\mathrm{CH}_{2} \mathrm{Cl}_{2}: \mathrm{MeOH}=20: 1$ was further purified by HPLC (YMC-Pack ODSA $_{18} ; 5 \mu \mathrm{m}, 250 \times 10 \mathrm{~mm}$, gradient elution $0-3 \mathrm{~min}, 74 \% \mathrm{ACN}$; $3-35 \mathrm{~min}$, 74-100\% ACN; $35.1-42 \mathrm{~min} 100 \% \mathrm{ACN})$ to yield $\mathbf{1 e}(7 \mathrm{mg})$ at $12.5 \mathrm{~min}, \mathbf{1 f}(12 \mathrm{mg})$ at $18.5 \mathrm{~min}, \mathbf{1 g}(12 \mathrm{mg})$ at $26.5 \mathrm{~min}$, and $1 \mathrm{~h}(12 \mathrm{mg})$ at $36 \mathrm{~min}$, respectively. The NMR data were summarized in Supplementary Tables 1, 2, and the NMR spectra were shown in Supplementary Figs. 21-28. For compounds 7b and 7c (Fig. 3a, b), the extract of $12 \mathrm{~L}$ culture of mutants Red $\alpha \beta 7029 \mathrm{glpCCs::genta} \mathrm{glbFCs::P_{Tn5-km } -}$ km-apra-holACs $(7029 \mathrm{H} \Delta \mathrm{GP}$ for compound $7 \mathbf{b})$ and Red $\alpha \beta 7029 \mathrm{glpCCs}:$ :genta glbFCs:: $\mathrm{P}_{\mathrm{Tn} 5-\mathrm{km}}-\mathrm{km}$-apra-glpCCs $(7029 \mathrm{P} \Delta \mathrm{GP}$ for compound $7 \mathrm{c})$, were eluted by a normal-phase silica gel column, respectively. For compound $7 \mathbf{b}$, gradient elution resulting in 9 fraction of $\mathrm{CH}_{2} \mathrm{Cl}_{2}: \mathrm{MeOH}=8: 1$ which was further purified by HPLC (YMC-Pack ODS-A C $18 ; 5 \mu \mathrm{m}, 250 \times 10 \mathrm{~mm}$, gradient elution 0-5 min, 30\% ACN; 5-25 $\mathrm{min}, 30-55 \% \mathrm{ACN} ; 25.1-35 \mathrm{~min} 95 \% \mathrm{ACN})$ to yield compound $7 \mathbf{b}(11 \mathrm{mg})$ at $21 \mathrm{~min}$. For compound $7 \mathbf{c}$, gradient elution resulting in 9 fraction of $\mathrm{CH}_{2} \mathrm{Cl}_{2}$ : $\mathrm{MeOH}=8: 1$ which was further purified by HPLC (YMC-Pack ODS-A C $\mathrm{C}_{18} ; 5 \mu \mathrm{m}$, $250 \times 10 \mathrm{~mm}$, gradient elution $0-5 \mathrm{~min}, 35 \% \mathrm{ACN}$; $5-25 \mathrm{~min}, 35-55 \% \mathrm{ACN}$; $25.1-35 \mathrm{~min} 95 \% \mathrm{ACN})$ to yield compound $7 \mathrm{c}(11 \mathrm{mg})$ at $21 \mathrm{~min}$. The NMR data of $7 \mathbf{b}$ and $7 \mathbf{c}$ were summarized in Supplementary Table 4 and the NMR spectra were shown in Supplementary Figs. 29-32.

Compound characterization. $\mathbf{1 d}, \mathbf{1 e}, \mathbf{1 f}, \mathbf{1 g}, \mathbf{1 h}, \mathbf{2 a}, \mathbf{7 b}$, and $7 \mathrm{c}:{ }^{1} \mathrm{H}$ NMR and ${ }^{13} \mathrm{C}$ NMR were summarized in Supplementary Tables 1-4; For 1d, UV: $\lambda_{\max } 224 \mathrm{~nm}$; HRMS $(m / z):[\mathrm{M}+\mathrm{H}]^{+}$calcd. for $\mathrm{C}_{41} \mathrm{H}_{65} \mathrm{~N}_{7} \mathrm{O}_{10}$, 816.4826; found, 816.4832; For 1e, UV: $\lambda_{\max } 227 \mathrm{~nm}$; HRMS $(\mathrm{m} / \mathrm{z}):[\mathrm{M}+\mathrm{H}]^{+}$calcd. for $\mathrm{C}_{43} \mathrm{H}_{69} \mathrm{~N}_{7} \mathrm{O}_{10}, 844.5139$; found, 844.5132; For 1f, UV: $\lambda_{\max } 228 \mathrm{~nm}$; HRMS $(m / z)$ : $[\mathrm{M}+\mathrm{H}]^{+}$calcd. for $\mathrm{C}_{45} \mathrm{H}_{73} \mathrm{~N}_{7} \mathrm{O}_{10}, 872.5452$; found, 872.5429; For 1g, UV: $\lambda_{\max } 228 \mathrm{~nm}$; HRMS $(m / z)$ : $[\mathrm{M}+\mathrm{H}]^{+}$calcd. for $\mathrm{C}_{47} \mathrm{H}_{77} \mathrm{~N}_{7} \mathrm{O}_{10}, 900.5765$; found, 900.5741; For 1h, UV: $\lambda_{\max }$ $228 \mathrm{~nm}$; HRMS $(\mathrm{m} / z)$ : $[\mathrm{M}+\mathrm{H}]^{+}$calcd. for $\mathrm{C}_{49} \mathrm{H}_{81} \mathrm{~N}_{7} \mathrm{O}_{10}, 928.6078$; found, 928.6070; For 2a, UV: $\lambda_{\max } 223 \mathrm{~nm}$; HRMS $(\mathrm{m} / z):[\mathrm{M}+\mathrm{H}]^{+}$calcd. for $\mathrm{C}_{36} \mathrm{H}_{56} \mathrm{~N}_{6} \mathrm{O}_{10}, 733.4091$; found, 733.4117; For 7b, UV: $\lambda_{\max } 235 \mathrm{~nm}$; HRMS $(\mathrm{m} / \mathrm{z})$ $[\mathrm{M}+\mathrm{H}]^{+}$calcd. for $\mathrm{C}_{23} \mathrm{H}_{40} \mathrm{~N}_{4} \mathrm{O}_{6}, 469.2981$; found, 469.3007; For 7c, UV: $\lambda_{\max } 235$ nm; HRMS $(\mathrm{m} / \mathrm{z})$ : $[\mathrm{M}+\mathrm{H}]^{+}$calcd. for $\mathrm{C}_{25} \mathrm{H}_{44} \mathrm{~N}_{4} \mathrm{O}_{6}, 497.3294$; found, 497.3304 .

Bioinformatic analysis. The sequence alignment of Cs domains was conducted by ClustalW2 and then processed by ESPript 3.0 $0^{53,54}$. The structures of Cs domains were predicted by SWISS-MODEL with tunnels calculated by Mole $2.0^{55,56}$.

Synthesis and purification of aminoacyl-SNACs. Aminoacyl-N-acetylcysteamine thioesters (SNACs) were synthesized as imitative substrates by the following method reported previously ${ }^{57}$. L-Leu-SNAC (4) and L-Val-SNAC (6) were purified by semipreparative RP-HPLC (column: YMC-Pack ODS-A C $18 ; 5 \mu \mathrm{m}, 250 \times$ $10 \mathrm{~mm}$, gradient elution: 0-5 min, $2 \% \mathrm{MeOH}$; $5-25 \mathrm{~min}, 2-30 \% \mathrm{MeOH} ; 25.1-35$ min, $95 \% \mathrm{MeOH}$; $35-40 \mathrm{~min}, 2 \% \mathrm{MeOH})$ to yield L-Leu-SNAC (4, $180 \mathrm{mg})$ at retention time $\sim 15.2 \mathrm{~min}$ and L-Val-SNAC $(\mathbf{6}, 230 \mathrm{mg})$ at retention time $\sim 15.0$ min, respectively. L-Ala-SNAC and L-Met-SNAC were purified by gradient elution (0-5 min, 5\% MeOH; 5-25 min, 5-35\% MeOH; 25.1-35 min 95\% MeOH) to yield L-Ala-SNAC at $\sim 10.5 \mathrm{~min}$ and L-Met-SNAC at $\sim 23.5 \mathrm{~min}$, respectively. L-PheSNAC was purified by gradient elution (0-5 min, $25 \% \mathrm{MeOH} ; 5-25 \mathrm{~min}, 25-40 \%$ $\mathrm{MeOH}$; $25.1-35 \mathrm{~min}, 95 \% \mathrm{MeOH})$ to yield at $\sim 17.8 \mathrm{~min}$. L-Ser-SNAC was purified by gradient elution ( $0-4 \mathrm{~min}, 5 \% \mathrm{MeOH}$; $4-22 \mathrm{~min}, 5-16 \% \mathrm{MeOH} ; 22.1-30 \mathrm{~min}$, $100 \% \mathrm{MeOH})$ at $\sim 13 \mathrm{~min}$. L-Thr-SNAC was purified by gradient elution $(0-4 \mathrm{~min}$, $8 \% \mathrm{MeOH}$; 4-22 $\min , 8-16 \% \mathrm{MeOH}$; $22.1-30 \mathrm{~min}, 100 \% \mathrm{MeOH}$ ) at $\sim 11 \mathrm{~min}$. 
Protein expression and purification. RzmA-Cs (NCBI: WP_013428324.1, residues 1-431), HolA-Cs (NCBI: WP 041754829.1, residues 1-431), and GlbF-Cs (NCBI: AKJ29077.1, residues 1-458) encoding regions were amplified by PCR and cloned into pET28a $(+)$ vector by LLHR of recombineering ${ }^{46}$. The correct plasmids were electroporated into E. coli BL21 (DE3) for protein expression. The $40 \mathrm{ml}$ overnight culture was inoculated into $2 \mathrm{~L} \mathrm{LB}$ medium supplemented with $5 \mu \mathrm{g} / \mathrm{ml}$ kanamycin and cultivated for $2.5 \mathrm{~h}$ at $37^{\circ} \mathrm{C}, 200 \mathrm{rpm}$. Then the bacteria were induced overnight with $0.1 \mathrm{mM}$ isopropyl-1-thio- $\beta$-D-galactopyranoside (IPTG) after precooled for $3 \mathrm{~h}$ at $18^{\circ} \mathrm{C}, 200 \mathrm{rpm}$. E. coli cells expressing RzmA-Cs, HolA-Cs, and GlbF-Cs were harvested and resuspended into $80 \mathrm{ml}$ wash buffer $(50 \mathrm{mM}$ Tris- $\mathrm{HCl}, 300 \mathrm{mM}$ $\mathrm{NaCl}$, and $50 \mathrm{mM}$ imidazole, $\mathrm{pH}=7.5$ ), and further lysed by sonication, respectively. Debris was removed by centrifugation for $1 \mathrm{~h}$ at $23,447 \times g$ before the supernatant being applied onto Ni-NTA agarose (Bestchrom). N-terminal hexahistidine-tagged proteins were digested with $140 \mathrm{U}$ thrombin enzyme (Solarbio) for $14 \mathrm{~h}$ at $4{ }^{\circ} \mathrm{C}$ after washed out impurity proteins as possible with $200 \mathrm{ml}$ wash buffer and balanced with analysis buffer $(50 \mathrm{mM}$ Tris- $\mathrm{HCl}, 300 \mathrm{mM} \mathrm{NaCl}, \mathrm{pH}=7.5)$ Tag-removed proteins were collected and concentrated in Amicon ${ }^{\circledR}$ Ultra-15 $30 \mathrm{~K}$ device (Millipore) before applied into size-exclusion chromatography by using the ÄKTA prime PLUS system (GE) mount with Superdex 200 pg 16/600 column. All proteins were purified in similar method.

Enzymatic activity assay. The purified Cs domain and mutants were subjected to enzyme activity assay. The substrates including aminoacyl-SNACs were stocked at $-80^{\circ} \mathrm{C}$ in $3 \%$ trifluoroacetic acid, the acyl-CoAs including acetyl-CoA (C2-CoA), butanoyl-CoA (C4-CoA), hexanoyl-CoA (C6-CoA), octanoyl-CoA (C8-CoA), decanoyl-CoA (C10-CoA), dodecanoyl-CoA (C12-CoA), myristoyl CoA (C14$\mathrm{CoA})$, palmitoyl-CoA (C16-CoA), and stearoyl-CoA (C18-CoA) were purchased from Sigma-Aldrich. For single assay, $20 \mu \mathrm{M}$ protein was mixed with $8 \mathrm{mM}$ aminoacyl-SNAC and $80 \mu \mathrm{M}$ acyl-CoA in the reaction buffer $(50 \mathrm{mM}$ Tris- $\mathrm{HCl}$, $300 \mathrm{mM} \mathrm{NaCl}, 1 \mathrm{mM}$ DTT and $20 \%$ glycerol, $\mathrm{pH}=7.5$ ) to final volume of $20 \mu \mathrm{l}$. The mixture was incubated at $30^{\circ} \mathrm{C}$ for $100 \mathrm{~min}$, and reaction was quenched with $20 \mu \mathrm{L} \mathrm{MeOH}$. The resulting mixture was centrifugated to remove the protein and $2 \mu \mathrm{l}$ of supernatant was used for UPLC-MS analysis. For the substrate competition assay, we used the mixture of $80 \mu \mathrm{M}$ of each acyl-CoA as donor substrates to investigate the specificities of RzmA-Cs and its variants for acyl-CoAs. The relative yield of each product was determined by comparison of its peak area in the UPLCMS chromatogram. The H140V/R148A mutant was tested by similar procedure in a series of time points, $0 \mathrm{~min}, 100 \mathrm{~min}, 300 \mathrm{~min}, 10 \mathrm{~h}$, and $24 \mathrm{~h}$, with modified substrates and protein concentrations (100 mM protein, $80 \mathrm{mM} \mathrm{L}$-Leu-SNAC, and $2 \mathrm{mM}$ C8-CoA). All tests were conducted in triplicates.

For the competition assay which simulates in vivo conditions, longer incubation time and different portions of acyl-CoAs were used. The $1.5 \mathrm{mM}$ acetyl-CoA plus $150 \mu \mathrm{M}$ butanoyl-CoA and $1.5 \mu \mathrm{M}$ others acyl-CoAs (from C6-CoA to C18-CoA) were mixed with $8 \mathrm{mM}$ L-Leu-SNAC and $20 \mu \mathrm{M}$ protein in reaction buffer for incubated $6 \mathrm{~h}$ at $30^{\circ} \mathrm{C}$ as described above. Extraction and analysis methods were same as described above. All tests were conducted with three replicates.

Four Cs domains were chosen to tested specificities of acceptor substrates, including RzmA-Cs, HolA-Cs, and GlbF-Cs. Seven different L-aminoacyl-SNACs including L-Val-SNAC, L-Leu-SNAC, L-Ala-SNAC, L-Phe-SNAC, L-Met-SNAC, L-Ser-SNAC, and L-Thr-SNAC were used as mimic acceptor substrates, and were checked with respective donor substrates, i.e., RzmA-Cs was tested with acetylCoA; HolA-Cs was tested with octanoyl-CoA and GlbF-Cs was checked with myristoyl-CoA by following procedure ${ }^{14}$. Twenty micromolar of proteins were mixed with $5 \mathrm{mM}$ aminoacyl-SNAC and $160 \mu \mathrm{M}$ acyl-CoA in the reaction buffer as described above. After incubation at $30^{\circ} \mathrm{C}$ for $120 \mathrm{~min}$, mixtures were extracted and analyzed as mentioned above. The relative yield of each product was determined by comparison of its peak area in the UPLC-MS chromatogram and the native product of mimic substrates were used as references, i.e., as the $100 \%$. All tests were conducted in triplicates.

Protein thermal shift assay. The purified protein was dissolved in analyze buffer ( $50 \mathrm{mM}$ Tris- $\mathrm{HCl}, 300 \mathrm{mM} \mathrm{NaCl}, \mathrm{pH}=7.5$ ) to reach the final concentration $4 \mu \mathrm{M}$, and tested by Protein Thermal Shift Dye Kit (ABI Lot: 00710602). The reaction mixture $(20 \mu \mathrm{l})$ was operated in Applied Biosystems Quant Studio ${ }^{\mathrm{TM}} 3$ Real-Time PCR System (Applied Biosystems Inc., CA, USA) in the condition as follows: step 1: $25^{\circ} \mathrm{C}, 1.6^{\circ} \mathrm{C} / \mathrm{s}$ and step $2: 99^{\circ} \mathrm{C}, 0.05^{\circ} \mathrm{C} / \mathrm{s}$ in the setting of excitation filter: $\mathrm{x} 4$ $(580 \pm 10 \mathrm{~nm})$ and emission filter: $\mathrm{m} 4(623 \pm 14 \mathrm{~nm})$. Melting curve data were analyzed by Protein Thermal Shift Software V1.30 (Applied Biosystems Inc., CA, USA) and $\Delta$ Tm (Boltzmann equation) was calculated.

Isothermal titration calorimetry. The purified RzmA-Cs R148A protein was dialyzed in analyze buffer $(50 \mathrm{mM}$ Tris- $\mathrm{HCl}, 100 \mathrm{mM} \mathrm{NaCl}, \mathrm{pH}=7.5)$ overnight. C8CoA was dissolved in a dialysis buffer. The binding affinity between C8-CoA and R148A was determined using ITC on PEAQ-ITC (Malvern). C8-CoA at $400 \mu \mathrm{M}$ was titrated into $400 \mu \mathrm{M}$ of R148A in the cell in dialysis buffer consisting of $50 \mathrm{mM}$ Tris- $\mathrm{HCl}, 100 \mathrm{mM} \mathrm{NaCl}, \mathrm{pH}=7.5$.

Crystallization and X-ray data collection. Crystallization of RzmA-Cs WT, R148A, and H140V/R148A proteins in the unbound form was carried out using sitting drop vapor diffusion method at $16^{\circ} \mathrm{C}$, by mixing equal volume of protein
$(10 \mathrm{mg} / \mathrm{ml})$ and the reservoir containing $30 \mathrm{mM}$ citric acid, $70 \mathrm{mM}$ Bis-Tris propane ( $\mathrm{pH}$ 7.6), and 20-25\% PEG 3350. The crystals of all co-complex and the "product-released" form were grown in the same precipitant solution of $100 \mathrm{mM}$ Bis-Tris propane ( $\mathrm{pH} 9.0)$ and 30-35\% PEG 6000 using hanging drop vapor diffusion method at $16^{\circ} \mathrm{C}$. Crystals of binary complex were obtained by R148A and H140V/R148A (10 mg/ml), respectively, co-crystallized with $2 \mathrm{mM} \mathrm{C8-CoA.}$ Crystal of ternary complex was obtained by H140V/R148A $(10 \mathrm{mg} / \mathrm{ml})$ cocrystallized with $2 \mathrm{mM} \mathrm{C8-CoA}$ and $5 \mathrm{mM}$ L-Leu-SNAC in 3 days, and ligand-free crystals in "product-released" status were obtained at the same drop in 2 weeks. Crystals were flash-frozen directly from the growing drops without additional cryoprotectants. Diffraction data of R148A + C8-CoA was collected at $100 \mathrm{~K}$ on beamlines BL17U1 at the Shanghai Synchrotron Radiation Facility (SSRF) ${ }^{58}$, and processed using XDS package ${ }^{59}$. The other diffraction data were collected on beamlines BL19U1 at the SSRF ${ }^{6}$. The data of RzmA-Cs WT, R148A, H140V/ R148A, and H140V/R148A + C8-CoA+Leu-SNAC were processed using the HKL2000 program $^{61}$. The data of H140V/R148A + C8-CoA and H140V/R148A product-released were processed using the HKL3000 program $^{62}$.

Structure determination and refinement. The structure of RzmA-Cs WT was solved by molecular replacement with the program Phaser ${ }^{63}$, using the CdaPS1-Cs (PDB code: 4 jn 3 ) structure as the search model ${ }^{18}$. Further manual model building was facilitated by using $\mathrm{Coot}^{64}$, combined with the structure refinement using Phenix ${ }^{65}$. Likewise, the other structures were solved by molecular replacement using RzmACs WT as the search model and further refined. Data collection and structure refinement statistics are summarized in Supplementary Table 5. The Ramachandran statistics, as calculated by Molprobity 66 , are $97 \% / 0.4 \%, 95 \% / 0.4 \%, 97 \% / 0 \%$, $96 \% / 0.3 \%, 99 \% / 0 \%, 97 \% / 0.2 \%$, and $99 \% / 0.2 \%$ (favored/outliers) for structures of RzmA-Cs WT, R148A, R148A + C8-CoA, H140V/R148A, H140V/R148A + C8CoA, H140V/R148A + C8-CoA+Leu-SNAC, and H140V/R148A productreleased, respectively. All the structural figures and the video were prepared using PyMol (The PyMol Molecular Graphics System, Version 2.3, Schrödinger, LLC).

Reporting summary. Further information on research design is available in the Nature Research Reporting Summary linked to this article.

\section{Data availability}

The data that support the findings of this study are available within the paper and its Supplementary Information files. A reporting summary for this article is available as a Supplementary Information file. The bacteria materials generated during the current study are available from the corresponding author upon reasonable request. Coordinates and structure data that support the findings of this study have been deposited in the Protein Data Bank with the accession codes 7C1H, 7C1K, 7C1L, 7C1P, 7C1R, 7C1S, and 7C1U (Supplementary Table 5). Source data are provided with this paper.

Received: 16 June 2020; Accepted: 2 December 2020; Published online: 12 January 2021

\section{References}

1. Schwarzer, D., Finking, R. \& Marahiel, M. A. Nonribosomal peptides: from genes to products. Nat. Prod. Rep. 20, 275-287 (2003).

2. Süssmuth, R. D. \& Mainz, A. Nonribosomal peptide synthesis-principles and prospects. Angew. Chem. Int. Ed. 56, 3770-3821 (2017).

3. Fischbach, M. A. \& Walsh, C. T. Assembly-line enzymology for polyketide and nonribosomal Peptide antibiotics: logic, machinery, and mechanisms. Chem. Rev. 106, 3468-3496 (2006).

4. Baltz, R. H., Miao, V. \& Wrigley, S. K. Natural products to drugs: daptomycin and related lipopeptide antibiotics. Nat. Prod. Rep. 22, 717-741 (2005).

5. Gotze, S. \& Stallforth, P. Structure, properties, and biological functions of nonribosomal lipopeptides from pseudomonads. Nat. Prod. Rep. 37, 29-54 (2020).

6. Pirri, G., Giuliani, A., Nicoletto, S. F., Pizzuto, L. \& Rinaldi, A. C. Lipopeptides as anti-infectives: a practical perspective. Cent. Eur. J. Biol. 4, 258-273 (2009).

7. Cochrane, S. A. \& Vederas, J. C. Lipopeptides from Bacillus and Paenibacillus spp.: a gold mine of antibiotic candidates. Med. Res. Rev. 36, 4-31 (2016).

8. Roongsawang, N., Washio, K. \& Morikawa, M. Diversity of nonribosomal peptide synthetases involved in the biosynthesis of lipopeptide biosurfactants. Int. J. Mol. Sci. 12, 141-172 (2010).

9. Raaijmakers, J. M., De Bruijn, I., Nybroe, O. \& Ongena, M. Natural functions of lipopeptides from Bacillus and Pseudomonas: more than surfactants and antibiotics. FEMS Microbiol. Rev. 34, 1037-1062 (2010).

10. Chooi, Y. H. \& Tang, Y. Adding the lipo to lipopeptides: do more with less. Chem. Biol. 17, 791-793 (2010).

11. Kraas, F. I., Giessen, T. W. \& Marahiel, M. A. Exploring the mechanism of lipid transfer during biosynthesis of the acidic lipopeptide antibiotic CDA. FEBS Lett. 586, 283-288 (2012). 
12. Liu, Q., Fan, W., Zhao, Y., Deng, Z. \& Feng, Y. Probing and engineering the fatty acyl substrate selectivity of starter condensation domains of nonribosomal peptide synthetases in lipopeptide biosynthesis. Biotechnol. J. 15, 1900175 (2020).

13. Kraas, F. I., Helmetag, V., Wittmann, M., Strieker, M. \& Marahiel, M. A. Functional dissection of surfactin synthetase initiation module reveals insights into the mechanism of lipoinitiation. Chem. Biol. 17, 872-880 (2010).

14. Imker, H. J., Krahn, D., Clerc, J., Kaiser, M. \& Walsh, C. T. N-acylation during glidobactin biosynthesis by the tridomain nonribosomal peptide synthetase module GlbF. Chem. Biol. 17, 1077-1083 (2010).

15. Debono, M. et al. Enzymatic and chemical modifications of lipopeptide antibiotic A21978C: the synthesis and evaluation of daptomycin (LY146032). J. Antibiot. 41, 1093-1105 (1988).

16. Oka, M. et al. Chemical modification of the antitumor antibiotic glidobactin. J. Antibiot. 41, 1812-1822 (1988).

17. Cochrane, S. A. et al. Synthesis and structure-activity relationship studies of $\mathrm{N}$-terminal analogues of the antimicrobial peptide tridecaptin A(1). J. Med. Chem. 57, 1127-1131 (2014).

18. Bloudoff, K., Rodionov, D. \& Schmeing, T. M. Crystal structures of the first condensation domain of CDA synthetase suggest conformational changes during the synthetic cycle of nonribosomal peptide synthetases. J. Mol. Biol. 425, 3137-3150 (2013).

19. Bloudoff, K., Alonzo, D. A. \& Schmeing, T. M. Chemical probes allow structural insight into the condensation reaction of nonribosomal peptide synthetases. Cell Chem. Biol. 23, 331-339 (2016).

20. Keating, T. A., Marshall, C. G., Walsh, C. T. \& Keating, A. E. The structure of VibH represents nonribosomal peptide synthetase condensation, cyclization and epimerization domains. Nat. Struct. Biol. 9, 522-526 (2002).

21. Drake, E. J. et al. Structures of two distinct conformations of holo-nonribosomal peptide synthetases. Nature 529, 235-238 (2016).

22. Kreitler, D. F., Gemmell, E. M., Schaffer, J. E., Wencewicz, T. A. \& Gulick, A. M. The structural basis of $\mathrm{N}$-acyl-alpha-amino-beta-lactone formation catalyzed by a nonribosomal peptide synthetase. Nat. Commun. 10, 3432 (2019).

23. Reimer, J. M., Aloise, M. N., Harrison, P. M. \& Schmeing, T. M. Synthetic cycle of the initiation module of a formylating nonribosomal peptide synthetase. Nature 529, 239-242 (2016).

24. Reimer, J. M. et al. Structures of a dimodular nonribosomal peptide synthetase reveal conformational flexibility. Science 366, eaaw4388 (2019).

25. Samel, S. A., Schoenafinger, G., Knappe, T. A., Marahiel, M. A. \& Essen, L. O. Structural and functional insights into a peptide bond-forming bidomain from a nonribosomal peptide synthetase. Structure 15, 781-792 (2007)

26. Tanovic, A., Samel, S. A., Essen, L. O. \& Marahiel, M. A. Crystal structure of the termination module of a nonribosomal peptide synthetase. Science 321, 659-663 (2008).

27. Tarry, M. J., Haque, A. S., Bui, K. H. \& Schmeing, T. M. X-ray crystallography and electron microscopy of cross- and multi-module nonribosomal peptide synthetase proteins reveal a flexible architecture. Structure 25, 783-793 (2017).

28. Miller, B. R., Drake, E. J., Shi, C., Aldrich, C. C. \& Gulick, A. M. Structures of a nonribosomal peptide synthetase module bound to $\mathrm{MbtH}$-like proteins support a highly dynamic domain architecture. J. Biol. Chem. 291, 22559-22571 (2016).

29. Wang, X. et al. Discovery of recombinases enables genome mining of cryptic biosynthetic gene clusters in Burkholderiales species. Proc. Natl Acad. Sci. USA 115, E4255-E4263 (2018).

30. Chen, H. et al. Identification of holrhizins E-Q reveals the diversity of nonribosomal lipopeptides in Paraburkholderia rhizoxinica. J. Nat. Prod. 83, 537-541 (2020).

31. Niehs, S. P., Scherlach, K. \& Hertweck, C. Genomics-driven discovery of a linear lipopeptide promoting host colonization by endofungal bacteria. Org. Biomol. Chem. 16, 8345-8352 (2018).

32. Ehmann, D. E., Trauger, J. W., Stachelhaus, T. \& Walsh, C. T. AminoacylSNACs as small-molecule substrates for the condensation domains of nonribosomal peptide synthetases. Chem. Biol. 7, 765-772 (2000).

33. Bian, X. et al. Heterologous production and yield improvement of epothilones in burkholderiales strain DSM 7029. ACS Chem. Biol. 12, 1805-1812 (2017).

34. Tang, B. et al. Reclassification of 'Polyangium brachysporum' DSM 7029 as Schlegelella brevitalea sp. nov. Int. J. Syst. Evolut. Microbiol. 69, 2877-2883 (2019).

35. Schellenberg, B., Bigler, L. \& Dudler, R. Identification of genes involved in the biosynthesis of the cytotoxic compound glidobactin from a soil bacterium. Environ. Microbiol. 9, 1640-1650 (2007).

36. Calcott, M. J., Owen, J. G. \& Ackerley, D. F. Efficient rational modification of non-ribosomal peptides by adenylation domain substitution. Nat. Commun. 11, 4554 (2020).

37. Stachelhaus, T., Mootz, H. D., Bergendahl, V. \& Marahiel, M. A. Peptide bond formation in nonribosomal peptide biosynthesis. Catalytic role of the condensation domain. J. Biol. Chem. 273, 22773-22781 (1998).
38. Bozhuyuk, K. A. J. et al. De novo design and engineering of non-ribosomal peptide synthetases. Nat. Chem. 10, 275-281 (2018).

39. Bloudoff, K. \& Schmeing, T. M. Structural and functional aspects of the nonribosomal peptide synthetase condensation domain superfamily: discovery, dissection and diversity. Biochim. Biophys. Acta Proteins Proteom. 1865, 1587-1604 (2017).

40. Ines, M. \& Dhouha, G. Lipopeptide surfactants: production, recovery and pore forming capacity. Peptides 71, 100-112 (2015).

41. Lewis, R. A. et al. Active site modification of the beta-ketoacyl-ACP synthase FabF3 of Streptomyces coelicolor affects the fatty acid chain length of the CDA lipopeptides. Chem. Commun. 47, 1860-1862 (2011).

42. Chen, L. et al. Engineering of new pneumocandin side-chain analogues from glarea lozoyensis by mutasynthesis and evaluation of their antifungal activity. ACS Chem. Biol. 11, 2724-2733 (2016).

43. Bozhuyuk, K. A. J. et al. Modification and de novo design of non-ribosomal peptide synthetases using specific assembly points within condensation domains. Nat. Chem. 11, 653-661 (2019).

44. Bennett, B. D. et al. Absolute metabolite concentrations and implied enzyme active site occupancy in Escherichia coli. Nat. Chem. Biol. 5, 593-599 (2009).

45. Chen, W. H., Li, K., Guntaka, N. S. \& Bruner, S. D. Interdomain and intermodule organization in epimerization domain containing nonribosomal peptide synthetases. ACS Chem. Biol. 11, 2293-2303 (2016).

46. Fu, J. et al. Full-length RecE enhances linear-linear homologous recombination and facilitates direct cloning for bioprospecting. Nat. Biotechnol. 30, 440-446 (2012).

47. Wang, H. et al. RecET direct cloning and Redalphabeta recombineering of biosynthetic gene clusters, large operons or single genes for heterologous expression. Nat. Protoc. 11, 1175-1190 (2016).

48. Wang, H. et al. ExoCET: exonuclease in vitro assembly combined with RecET recombination for highly efficient direct DNA cloning from complex genomes. Nucleic Acids Res. 46, e28 (2018).

49. Tang, B., Yu, Y., Zhang, Y., Zhao, G. \& Ding, X. Complete genome sequence of the glidobactin producing strain [Polyangium] brachysporum DSM 7029. J. Biotechnol. 210, 83-84 (2015).

50. Bian, X., Plaza, A., Yan, F., Zhang, Y. \& Müller, R. Rational and efficient sitedirected mutagenesis of adenylation domain alters relative yields of luminmide derivatives in vivo. Biotechnol. Bioeng. 112, 1343-1353 (2015).

51. Wang, H. et al. Improved seamless mutagenesis by recombineering using $\mathrm{ccdB}$ for counterselection. Nucleic Acids Res. 42, e37 (2014).

52. Wang, Z. J. et al. Genome mining and biosynthesis of primary amine-acylated desferrioxamines in a marine gliding bacterium. Org. Lett. 22, 939-943 (2020).

53. Larkin, M. A. et al. Clustal W and Clustal X version 2.0. Bioinformatics 23, 2947-2948 (2007)

54. Robert, X. \& Gouet, P. Deciphering key features in protein structures with the new ENDscript server. Nucleic Acids Res. 42, W320-W324 (2014).

55. Sehnal, D. et al. MOLE 2.0: advanced approach for analysis of biomacromolecular channels. J. Cheminform. 5, 39 (2013).

56. Waterhouse, A. et al. SWISS-MODEL: homology modelling of protein structures and complexes. Nucleic Acids Res. 46, W296-W303 (2018).

57. Ehmann, D. E., Shaw-Reid, C. A., Losey, H. C. \& Walsh, C. T. The EntF and EntE adenylation domains of Escherichia coli enterobactin synthetase: sequestration and selectivity in acyl-AMP transfers to thiolation domain cosubstrates. Proc. Natl Acad. Sci. USA 97, 2509-2514 (2000).

58. Wang, Q. S. et al. Upgrade of macromolecular crystallography beamline BL17U1 at SSRF. Nucl. Sci. Tech. 29, 68 (2018).

59. Kabsch, W. XDS. Acta Crystallogr. D. Biol. Crystallogr. 66, 125-132 (2010).

60. Zhang, W. Z. et al. The protein complex crystallography beamline (BL19U1) at the Shanghai Synchrotron Radiation Facility. Nucl. Sci. Tech. 30, 170 (2019).

61. Otwinowski, Z. \& Minor, W. Processing of X-ray diffraction data collected in oscillation mode. Method Enzymol. 276, 307-326 (1997).

62. Minor, W., Cymborowski, M., Otwinowski, Z. \& Chruszcz, M. HKL-3000: the integration of data reduction and structure solution - from diffraction images to an initial model in minutes. Acta Crystallogr. D. Biol. Crystallogr. 62, 859-866 (2006)

63. Mccoy, A. J. et al. Phaser crystallographic software. J. Appl. Crystallogr 40, 658-674 (2007).

64. Emsley, P., Lohkamp, B., Scott, W. G. \& Cowtan, K. Features and development of Coot. Acta Crystallogr. D. Biol. Crystallogr. 66, 486-501 (2010).

65. Adams, P. D. et al. PHENIX: a comprehensive Python-based system for macromolecular structure solution. Acta Crystallogr. D. Biol. Crystallogr. 66, 213-221 (2010).

66. Chen, V. B. et al. MolProbity: all-atom structure validation for macromolecular crystallography. Acta Crystallogr. D. Biol. Crystallogr. 66, 12-21 (2010).

\section{Acknowledgements}

We would like to thank Zhifeng Li, Jingyao Qu, Xiaoju Li, and Haiyan Sui from State Key Laboratory of Microbial Technology of SDU for data collection of UPLC-HRMS, NMR, 
and X-ray diffraction, and Dr. V. Ravichandran (SDU) for proofreading of the manuscript. We also thank the staff from beamlines BL19U1 and BL17U1 at Shanghai Synchrotron Radiation Facility (SSRF) for assistance during data collection. This study was supported by the National Key R\&D Program of China (2019YFA0905700,

2017YFD0201400, 2018YFE0113000), the National Natural Science Foundation of China (31670098, 32070060, 31670097, 31700114), the Shandong Provincial Natural Science Foundation, China (ZR2019JQ11), the Natural Science Foundation of Jiangsu Province (BK20170399), the 111 project (B16030), the Taishan Scholars Program of Shandong Province (tsqn201909004), and the Qilu Young Scholar Startup Funding and Youth Interdisciplinary Innovative Research Group (2020QNQT009) of SDU.

\section{Author contributions}

X.B. and D.W. conceived the study; X.B., D.W., and Y.Z. supervised the experiments; L.Z., N.Z., H.C., H.Z., X.B, Y.Z., and X.B. constructed mutants and expressed plasmids, analyzed metabolic data and purified compounds, isolated the proteins, and conducted biochemical studies; L.Z., X.D., and N.Z. carried out crystallizations; X.D., F.L., X.R., and D.W. collected X-ray diffraction data, solved, and refined the structures; L.Z., X.D., D.W., and X.B. wrote the manuscript with the input from all authors.

\section{Competing interests}

The authors declare no competing interests.

\section{Additional information}

Supplementary information is available for this paper at https://doi.org/10.1038/s41467020-20548-8.

Correspondence and requests for materials should be addressed to Y.Z., D.W. or X.B.

Peer review information: Nature Communications thanks David Ackerley, Andrew Gulick and the other anonymous reviewers for their contribution to the peer review of this work. Peer reviewer reports are available.

Reprints and permission information is available at http://www.nature.com/reprints

Publisher's note Springer Nature remains neutral with regard to jurisdictional claims in published maps and institutional affiliations.

\section{(c) (i)} adaptation, distribution and reproduction in any medium or format, as long as you give appropriate credit to the original author(s) and the source, provide a link to the Creative Commons license, and indicate if changes were made. The images or other third party material in this article are included in the article's Creative Commons license, unless indicated otherwise in a credit line to the material. If material is not included in the article's Creative Commons license and your intended use is not permitted by statutory regulation or exceeds the permitted use, you will need to obtain permission directly from the copyright holder. To view a copy of this license, visit http://creativecommons.org/ licenses/by/4.0/.

(C) The Author(s) 2021 